\title{
EMBEDDINGS OF RIEMANNIAN MANIFOLDS WITH FINITE EIGENVECTOR FIELDS OF CONNECTION LAPLACIAN
}

\author{
CHEN-YUN LIN AND HAU-TIENG WU
}

\begin{abstract}
We study the problem asking if one can embed manifolds into finite dimensional Euclidean spaces by taking finite number of eigenvector fields of the connection Laplacian. This problem is essential for the dimension reduction problem in massive data analysis. Singer-Wu proposed the vector diffusion map which embeds manifolds into the Hilbert space $l^{2}$ using eigenvectors of connection Laplacian. In this paper, we provide a positive answer to the problem. Specifically, we use eigenvector fields to construct local coordinate charts with low distortion, and show that the distortion constants depend only on geometric properties of manifolds with metrics in the little Hölder space $c^{2, \alpha}$. Next, we use the coordinate charts to embed the entire manifold into a finite dimensional Euclidean space. The proof of the results relies on solving the elliptic system and provide estimates for eigenvector fields and the heat kernel and their gradients. We also provide approximation results for eigenvector field under the $c^{2, \alpha}$ perturbation.
\end{abstract}




\section{INTRODUCTION}

In the past decade, there is a growing consensus that high dimensional and massive dataset analysis is a key to future advances. Although frequently the information regarding the structure underlying a dataset is limited, we generally believe that a "lower dimensional" and possibly nonlinear structure should exist.

A widely accepted approach to model the low dimension structure is by considering the manifold; that is, we assume that the collected dataset, while might be of high dimension, is located on a low dimensional manifold [40, 5, 13. How to analyze the dataset under this assumption is generally called the manifold learning problem, and one particular goal is to recover the nonlinear low dimensional structure of the manifold. Under this assumption, several algorithms were proposed toward this goal, like isomap [40], locally linear embedding (LLE) [30], eigenmaps (EM) [5], diffusion maps (DM) [13], Hessian LLE [17], vector diffusion maps [33, 35], nonlinear independent component analysis or empirical intrinsic geometry [31, 38], alternating diffusion [23, 39], etc. In a nutshell, by taking the local information of the given point cloud, like geodesic distance, and by applying knowledge from the spectral geometry and index theory, we could obtain information of the manifold from different aspects, like the parametrization of the dataset or the topological feature of the dataset.

One particular interesting problem is how to guarantee the data visualization and/or dimensional reduction. Data visualization problem is asking if we could embed a given manifold into the three dimensional Euclidean space, so that we could visualize the dataset; dimension reduction problem is asking if we could embed a given manifold into a low dimensional Euclidean space, which dimension is smaller than that of the dataset. Mathematically, this problem is formulated as asking if it is possible to embed the manifold (hence the dataset) into a finite dimensional Euclidean space, even isometrically. The embedding problem was first positively answered by Whitney [43], and the isometrically embedding problem was first solved by Nash [27]. However, the approach by Nash, the implicit function theory, is not canonical and is not essentially feasible for data analysis. In Berard, Besson and Gallot's breakthrough paper [7], the spectral embedding idea was explored to answer this kind of problem. The main idea is embedding the Riemannian manifold by the eigenfunctions of the associated LaplaceBeltrami operator via studying the associated heat kernel behavior. We could show that the spectral embedding is a canonical embedding in the sense that it depends only on the eigenfunctions and eigenvalues, and the result is close to isometric with error of the order of the diffusion time. In [42], this embedding was further modified to an almost isometric embedding, with error up to any give finite order of the diffusion time, by applying the implicit function theory.

The spectral embedding idea is directly related to many manifold learning algorithms, like EM and DM. While these algorithms work, however, numerically we are able to obtain only finite eigenfunctions and eigenvalues in practice, while theoretically all the eigenfunctions and eigenvalues of the Laplace-Beltrami operator, which are countably infinite, are needed to study the spectral embedding. Thus, the next natural question we could ask is the possibility to embed the manifold by the finite eigenfunctions and eigenvalues. This question was positively answered separately by Bates [4] and Portegies 
[29]; that is, one is able to embed the manifold with finite eigenfunctions and eigenvalues. In Portegies, it is further shown that the embedding could be almost isometric with a prescribed error bound.

The work in Bates is essentially based on the local parametrization work reported in Johns, Maggioni and Schul [22, where the inherited oscillatory behavior of the eigenfunction is taken into account to guarantee that locally we could find finite eigenfunctions so that we could embed a local ball via these finite eigenfunctions with low distortion. What needs to be proved is that the embedding of different local balls will not intersect each other. These works fundamentally answer why EM and DM could work well in practice.

The spectral embedding mentioned above depends on the Laplace-Beltrami operator. The vector diffusion map (VDM) [33, 35], on the other hand, depends on the connection Laplacian associated with a possibly nontrivial bundle structure. In brief, the VDM with the diffusion time $t>0$ is defined by the eigenvector fields of the connection Laplacian by

$$
\begin{aligned}
V_{t}: M & \rightarrow \ell^{2} \\
x & \mapsto\left(e^{-\left(\lambda_{i}+\lambda_{j}\right) t / 2}\left\langle X_{i}, X_{j}\right\rangle\right)_{i, j=1}^{\infty},
\end{aligned}
$$

where $x \in M$ and $X_{i}$ is the $i$-th eigenvector field of the connection Laplacian associated with the eigenvalue $\lambda_{i}$. The basic properties of $V_{t}$ have been shown in [33, 35, 44]. For example, we could see that the VDM is an embedding, and it is close to an isometric embedding with error depending on the diffusion time. The VDM is originally motivated by studying the cryo electron microscope problem, in particular the class averaging algorithm [37, 21, 45]. In general, the essential goal of VDM is to integrate different kinds of local/partial information and the relationship between these pieces of local information in order to obtain the global information of the dataset; for example, the ptychographic imaging problem [25], the vector nonlocal mean/median, the orientability problem [32], etc. Numerically, the VDM depends on the spectral study of the graph connection Laplacian (GCL) [33, 12, 11, 35, 18, 19], which is a direct generalization of the graph Laplacian discussed in the spectral graph theory [10].

1.1. Our contribution. A fundamental problem regarding the VDM, like that in the spectral embedding, is that if we could embed the manifold with only finite eigenvector fields; that is, can we find a finite number $n \in \mathbb{N}$ so that the truncated VDM (tVDM), defined as

$$
\begin{aligned}
V_{t}^{N^{2}}: M & \rightarrow \mathbb{R}^{N^{2}} \\
x & \mapsto\left(e^{-\left(\lambda_{i}+\lambda_{j}\right) t / 2}\left\langle X_{i}, X_{j}\right\rangle(x)\right)_{i, j=1}^{n},
\end{aligned}
$$

is an embedding? In this paper, we provide a positive answer.

Theorem 1 (Embeddings of Riemannian Manifolds with Finite Eigenvector Fields of Connection Laplacian). For a smooth closed manifold $(M, g)$ with smooth metric and for any $t>0$, there is a positive integer $N_{0}$ so that the $t V D M V_{t}^{N^{2}}$ is a smooth embedding for all $N \geq N_{0}$. 
With the above theorems we could answer the raised question positively and say that with a proper chosen finite number of eigenvector fields, the tVDM is an embedding.

Denote a class of closed smooth manifolds of dimension $d$ by

$$
\mathcal{M}_{n, \kappa, i_{0}, V}=\left\{\left(M^{n}, g\right):|\operatorname{Ric}(g)| \leq \kappa, \operatorname{inj}(\mathrm{M}) \geq i_{0}, \operatorname{Vol}(M) \leq V\right\},
$$

where $\operatorname{inj}(M)$ denotes the injectivity radius of $M$.

Theorem 2 (Embeddings of Manifolds with $c^{2, \alpha}$ metrics). Take $0<\alpha \leq 1$. For $(M, g) \in \mathcal{M}_{n, \kappa, i_{0}, V}$ with $g \in c^{2, \alpha}$ so that $|R i c| \leq \kappa$, and for any $t>0$, there us a positive integer $N_{0}$ so that the $\operatorname{VDM} V_{t}^{N^{2}}$ is an embedding for all $N \geq N_{0}$.

Here $c^{k, \alpha}$ denotes the little Hölder space which is the closure of $C^{\infty}$ functions in the Hölder space $C^{k, \alpha}$.

Throughout the paper, we try to quantify all the bounds by $n, \kappa, i_{0}$, and $V$ in hope that we can embedding results for the whole class of manifolds $\mathcal{M}_{n, \kappa, i_{0}, V}$. However, we still lack of universal estimates for Lemma 10 for manifolds in $\mathcal{M}_{n, \kappa, i_{0}, V}$. If one, can obtain such universal bounds, then the following conjecture is proved as well.

Conjecture 3 (Embeddings of Riemannian Manifolds with Finite Eigenvector Fields of Connection Laplacian). There is a positive integer $N_{0}$ so that for all $(M, g) \in \mathcal{M}_{n, \kappa, i_{0}, V}$, $N \geq N_{0}$ and for any $t>0$, the $t V D M V_{t}^{N^{2}}$ is a smooth embedding.

1.2. Organization. The paper is organized in the following. In Section 2, we introduce our notation convention and provide some back ground material.

In Section 3, under the assumption that the metric is smooth, we provide necessary Lemma 10,13 for proving the universal local parametrization for any manifold in $\mathcal{M}_{d, \kappa, i_{0}, V}$

In Section 4, under the assumption that the metric is smooth, we show the global embedding result, Theorem 1. To show the proof, we provide an immersion result in Lemma 14 based on Theorem 5. Then, in Lemma 15 we control the remainder term of the series expansion of the Hilbert-Schmidt norm of the heat kernel associated with the connection Laplacian. In Section 5, we approximate a metric with low regularity by a smooth metric, and hence prove Theorem 1 under the assumption that the metric is of low regularity. In Section 6, we provide two examples to illustrate how VDM is carried out numerically.

Acknowledgements: Hau-tieng Wu's research is partially supported by Sloan Research Fellow FR-2015-65363. Chen-Yun Lin would like to thank Thomas Nyberg for his helpful discussions.

\section{Background Material and Notation}

Let $(M, g)$ be a smooth compact manifold of dimension $n$ without boundary with metric $g$. We assume that $g$ is smooth except the technical part (Lemmas 6, 7, Proposition 8, and Corollary 9 in Section 3 and Section 5 where we assume $g$ is $c^{2}, \alpha$. Denote $d_{g}(x, y)$ to be the geodesic distance between $x$ and $y$. Let $D$ denote the diameter of $(M, g)$.

Anderson [2] showed that the existence of harmonic coordinates on balls of uniform size is guaranteed by imposing suitable geometric conditions. The bounds $|R i c| \leq \kappa$ 


\begin{tabular}{|c|c|c|}
\hline & Symbol & Meaning \\
\hline Setup & $\begin{array}{l}n \\
M \\
g \\
T M \\
\langle\cdot, \cdot\rangle \\
d_{g}(\cdot, \cdot) \\
P_{x}^{y} \\
(\phi, U)\end{array}$ & $\begin{array}{l}\text { dimension of the Riemannian manifold } \\
\text { smooth closed Riemannian manifold } \\
\text { Riemannian metric } \\
\text { tangent bundle of } M \\
\text { inner product with respect } g \\
\text { geodesic distance } \\
\text { parallel transport from } x \text { to } y \\
\text { local (harmonic) coordinate chart }\end{array}$ \\
\hline Geometric Conditions & $\begin{array}{l}V \\
D \\
\kappa \\
i_{0}\end{array}$ & $\begin{array}{l}\text { volume upper bound } \\
\text { diameter upper bound } \\
\text { Ricci curvature bound, } \mid \text { Ric } \mid \leq \kappa \\
\text { injectivity radius lower bound }\end{array}$ \\
\hline Indices & $\begin{array}{l}a, b, c, \cdots \\
i, j, k, \cdots\end{array}$ & $\begin{array}{l}\text { indices for coordinate charts } \\
\text { indicies for the spectrum }\end{array}$ \\
\hline Embedding & $\begin{array}{l}N \\
\Phi_{z} \\
\tilde{\Phi}_{z} \\
\mu_{i j} \\
\Phi^{N^{2}}\end{array}$ & $\begin{array}{l}\text { dimension of the ambient Euclidean space } \\
\text { local parametrization of } M \\
\text { weighted local parametrization of } M \\
\text { associated weight with respect to } X_{i} \text { and } X_{j} \\
\text { embedding of } M \text { into } \mathbb{R}^{N^{2}}\end{array}$ \\
\hline Derivatives & $\begin{array}{l}\partial_{i} \\
\nabla_{g} \\
\Gamma_{a b}^{c} \\
\Delta_{g} \\
\nabla_{g}^{2} \\
\end{array}$ & $\begin{array}{l}\text { partial derivative w.r.t coordinate } x_{i} \\
\text { gradient/Levi-Civita connection of } g \\
\text { Christoffel symbols } \\
\text { Laplace-Beltrami operator } \\
\text { connection Laplacian }\end{array}$ \\
\hline Spectra & $\begin{array}{l}\lambda_{i} \\
X_{i} \\
\nu_{i} \\
\xi_{i}\end{array}$ & $\begin{array}{l}\text { spectrum of } \nabla^{2} \text {, counting multiplicity } \\
L^{2} \text {-normalized eigenvector fields, } \nabla^{2} X_{i}=-\lambda_{i} X_{i} \\
\text { spectrum of } \Delta \text {, counting multiplicity } \\
L^{2} \text {-normalized eigenfunctions, } \Delta \xi_{i}=-\nu_{i} \xi_{i}\end{array}$ \\
\hline Spaces & $\begin{array}{l}L^{p, \infty}(M, g) \\
c^{2, \alpha}\end{array}$ & $\begin{array}{l}\text { the weak } L^{p} \text { spaces } \\
\text { the little Hölder space }\end{array}$ \\
\hline Heat Kernels & $\begin{array}{l}k_{T M}(t, z, w) \\
k_{M}(t, z, w) \\
\nabla_{v} k_{T M}(t, \cdot \cdot \cdot) \\
\left\|k_{T M}(t, \cdot \cdot \cdot)\right\|_{H S}^{2} \\
\end{array}$ & $\begin{array}{l}\text { heat kernel of the heat semigroup } e^{-t \nabla^{2}} \\
\text { heat kernel of the heat semigroup } e^{-t \Delta} \\
\text { derivative w.r.t. the second spatial variable } \\
\text { Hilber-Schmidt norm of } k_{T M}(t, \cdot, \cdot)\end{array}$ \\
\hline Other & $\begin{array}{l}f_{1} \sim_{c}^{C} f_{2} \\
c \ll C\end{array}$ & $\begin{array}{l}c f_{2} \leq f_{1} \leq C f_{2} \\
c / C \text { is sufficiently small for positive values } c, C\end{array}$ \\
\hline
\end{tabular}

TABLE 1. Summary of symbols 
and $\operatorname{inj}(M) \geq i_{0}$ (or the volume bound) alone imply a lower bound on the size of balls $B$ on which one has harmonic coordinates with $C^{1, \alpha}$ bounds on the metric. Here, we restate Main Lemma 2.2 in [2] for our case.

Lemma. Let $(M, g)$ be a closed Riemannian n-manifold such that

$$
|R i c| \leq \kappa \quad \operatorname{inj}(M) \geq i_{0}
$$

Then given any $Q>1, \alpha \in(0,1)$, there exists an constant $\epsilon_{0}=\epsilon_{0}(Q, \kappa, n, \alpha)$ with the following property: given any point $z \in M$, there is a harmonic coordinate system $\phi: U \subset \mathbb{R}^{n} \rightarrow B_{r}(z), r \geq \epsilon_{0} i_{0}$ such that $\phi(0)=z, g_{a b}(0)=\delta_{a b}$, and

$$
\begin{array}{r}
Q^{-1} \delta_{a b} \leq g_{a b} \leq Q \delta_{a b} \text { on } U \\
r^{1+\alpha}\|g\|_{C^{1, \alpha}(U)} \leq Q .
\end{array}
$$

For any $z \in M$, let $(\phi, U), \phi: U \subset \mathbb{R}^{n} \rightarrow M$, be a harmonic coordinate chart so that $z=\phi(0) \in \phi(U), g_{a b}(0)=\delta_{a b}$, and $Q^{-1}<g<Q$ on $U$. Set

$$
R_{z}=\sup _{r}\left\{r>0: B_{r}(z) \subset \phi(U)\right\} .
$$

There are several advantages that we have using harmonic coordinates. First, we can obtain universal harmonic radius lower bound. Second, a metric has optimal regularity in harmonic coordinate charts.

Theorem. [15, Theorem 2.1] If a metric $g \in C^{k, \alpha}, 1 \leq k \leq \infty$ in some coordinates chart, then it is also of class $C^{k, \alpha}$ in harmonic coordinates, while it is of at least class $C^{k-2, \alpha}$ in geodesic normal coordinates.

Last, the connection Laplacian of a vector field simplifies in harmonic coordinates and can be expressed in terms of $g, \partial g$, and the Ricci curvature. Let $\left\{x^{a}\right\}$ be a harmonic coordinate system on $M$ and $X=X^{a} \frac{\partial}{\partial x^{a}}$ be a vector field.

Let $\nabla_{g}$ denote the Levi-Civita connection of $(M, g)$ and $\nabla_{g}^{2}$ the associated connection Laplacian [9] on the tangent bundle $T M$. Denote $\Delta_{g}$ to be the Laplace-Beltrami operator of $(M, g)$. When there is no danger of confusion, we will ignore the subscript $g$. Note that

$$
\Delta x^{c}=g^{a b} \Gamma_{a b}^{c}=0,
$$

and the Ricci curvature in the harmonic coordinates can be expressed as

$$
-\mathrm{Ric}_{d}^{c}=g^{a b} \partial_{a} \Gamma_{d b}^{c}+\partial_{m} g^{a b} \Gamma_{a b}^{c}+g^{a b} \Gamma_{d b}^{e} \Gamma_{a e}^{c} .
$$

Hence,

$$
\begin{aligned}
\nabla^{2}\left(X^{c} \partial_{c}\right) & =-g^{a b}\left(\partial_{a} \partial_{b} X^{c}+2 \Gamma_{b d}^{c} \partial_{a} X^{d}+\Gamma_{a e}^{c} \Gamma_{b d}^{e} X^{d}+\partial_{a} \Gamma_{b d}^{c} X^{d}\right) \partial_{c} \\
& =\left(-g^{a b} \partial_{a} \partial_{b} X^{c}-2 g^{a b} \Gamma_{b d}^{c} \partial_{a} X^{d}+\partial_{d} g^{a b} \Gamma_{a b}^{c} X^{d}+R c_{d}^{c} X^{d}\right) \partial_{c}
\end{aligned}
$$

and the coefficients of $\nabla^{2} X$ are controlled in $C^{1, \alpha}$ harmonic coordinates and the Ricci curvature bound.

It is known [20] that both $\nabla^{2}$ and $\Delta$ are self-adjoint, elliptic and that their spectra are discrete and non-positive real numbers with $-\infty$ as the only possible accumulating point. Furthermore, the eigenspaces are all finite dimensional. We denote the spectrum 
of $\nabla^{2}$ as $\left\{-\lambda_{i}\right\}_{i=1}^{\infty}$, where $0 \leq \lambda_{1} \leq \lambda_{2} \leq \cdots$, counting algebraic multiplicity, and denote the corresponding orthonormal basis of eigenvector fields for $L^{2}(T M)$ as $\left\{X_{i}\right\}_{i=1}^{\infty}$; that is, $\nabla^{2} X_{i}=-\lambda_{i} X_{i}$ for all $i=1,2 \cdots$ and

$$
\int_{M}\left\langle X_{i}, X_{j}\right\rangle d \sigma=\delta_{i j}
$$

where $d \sigma$ is the Riemannian measure associated with $g$. Note that $\lambda_{1}$ may or may not be 0 due to the topological constraint. Also denote the spectrum of $\Delta$ as $\left\{-\nu_{i}\right\}_{i=1}^{\infty}$, counting algebraic multiplicity, where $0=\nu_{1} \leq \nu_{2} \leq \cdots$ and denote the corresponding orthonormal basis of eigenfunctions for $L^{2}(M)$ as $\left\{\xi_{i}\right\}_{i=1}^{\infty}$. Note that compared with the connection Laplacian, $\nu_{1}$ is always 0 .

The heat semigroup is the family of self-adjoint operators $e^{-t \nabla^{2}}, t>0$, with a smooth heat kernel $k_{T M}(t, x, y)$, where $k_{T M}(t, x, y)$ is smooth in $x, y \in M$ and analytic in $t$ when $t>0$ [20]. More precisely, for any $X \in L^{2}(T M)$,

$$
e^{-t \nabla^{2}} X(x)=\int_{M} k_{T M}(t, x, y) X(y) d \sigma(y) .
$$

Given an $L^{2}(T M)$-orthonormal basis $\left\{X_{i}\right\}_{i=1}^{\infty}$ of the eigenvector fields, the heat kernel of $\nabla^{2}$ can be expressed as

$$
k_{T M}(t, x, y)=\sum_{i=1}^{\infty} e^{-\lambda_{i} t} X_{i}(x) \otimes X_{i}(y),
$$

where $t>0$ and $z, w \in M$. Its Hilbert-Schmidt norm is defined as

$$
\left\|k_{T M}(t, x, y)\right\|_{H S}^{2}=\operatorname{Tr}\left(k_{T M}(t, x, y)^{*} k_{T M}(t, x, y)\right) \text {. }
$$

A direct computation [33] shows that the Hilbert-Schmidt norm squared of the heat kernel can be written as the series

$$
\left\|k_{T M}(t, x, y)\right\|_{H S}^{2}=\sum_{i, j} e^{-\left(\lambda_{i}+\lambda_{j}\right) t}\left\langle X_{i}(x), X_{j}(x)\right\rangle\left\langle X_{i}(y), X_{j}(y)\right\rangle .
$$

Based on (2.11), the VDM (1.1) and tVDM (1.2) are proposed in [33]. Throughout the paper, we write $\nabla_{v}\left\|k_{T M}(t, \cdot, \cdot)\right\|_{H S}$ to denote the covariant derivative with respect to the second variable at time $t$.

In what follows, we use $c$ and $C$ to denote constant which may vary line by line. We write $f_{1} \sim_{c}^{C} f_{2}$ if there exist constants $c$ and $C$ such that $c f_{2} \leq f_{1} \leq C f_{2}$. For two positive values $c, C$, we write $c \ll C$ to denote that $c / C$ is sufficiently small.

\section{Universal Local Parametrization for Smooth Manifolds}

To embed a Riemannian manifold with finite eigenvector fields (Theorem 1), we first show in Theorem 5 that we could parametrize local balls of closed manifolds via eigenvector fields. While the proof strategy follows the ideas of [22], we provide several new estimates specific for the vector fields and connection Laplacian, which have their own independent interest.

Assumption 4. For $R \leq R_{z}$, choose $\delta_{1}>0$ so that $\delta_{1}^{2} R^{2} \ll 1$ and choose $\delta_{0}>0$ so that $\delta_{0} \ll \delta_{1}$. We consider $t$ satisfying $\frac{1}{2} \delta_{1}^{2} R^{2} \leq t \leq \delta_{1}^{2} R^{2}$ and $w, z \in B_{\delta_{0} R}(z) \backslash B_{\frac{1}{2} \delta_{0} R}(z)$. 
Theorem 5 (Parametrization via eigenvector fields for manifolds). Let $\left(M^{n}, g\right)$ be a smooth closed manifold with smooth metric $g$, Ricci curvature bound $\kappa$ and diameter upper bound D. Fix $z \in M$ and assume that Assumption 4 holds. For $R \leq R_{z}$, there exist a constant $\tau=\tau\left(n, Q, \kappa, i_{0}, \alpha, \delta_{1}\right)>1$, and $n$ pairs of indices $\left(i_{1}, j_{1}\right), \cdots,\left(i_{n}, j_{n}\right)$ so that the map

$$
\begin{aligned}
\Phi_{z}: B_{\tau^{-1} R}(z) & \rightarrow \mathbb{R}^{n} \\
x & \mapsto\left(\left\langle X_{i_{1}}, X_{j_{1}}\right\rangle(x), \cdots,\left\langle X_{i_{n}}, X_{j_{n}}\right\rangle(x)\right),
\end{aligned}
$$

is a parametrization of $B_{\tau^{-1} R}(z)$, where the associated eigenvalues satisfy

$$
\tau^{-1} R^{-2} \leq \lambda_{i_{1}}, \cdots, \lambda_{i_{n}}, \lambda_{j_{1}}, \cdots, \lambda_{j_{n}} \leq \tau R^{-2} .
$$

Furthermore, if $\Phi_{z}$ is weighted properly by

$$
\begin{aligned}
\tilde{\Phi}_{z}: B_{\tau^{-1} R}(z) & \rightarrow \mathbb{R}^{n} \\
x & \mapsto\left(\mu_{1}\left\langle X_{i_{1}}, X_{j_{1}}\right\rangle(x), \cdots, \mu_{n}\left\langle X_{i_{n}}, X_{j_{n}}\right\rangle(x)\right),
\end{aligned}
$$

where

$$
\mu_{k}:=\mu_{i_{k} j_{k}}=\left(f_{B_{\tau^{-1} R}(z)}\left\|X_{i_{k}}\right\|_{g}^{2}\right)^{-1 / 2}\left(f_{B_{\tau^{-1} R}(z)}\left\|X_{j_{k}}\right\|_{g}^{2}\right)^{-1 / 2},
$$

then for any $x, y \in B_{\tau^{-1} R}(z)$, we have

$$
\frac{1}{\tau R} d_{g}(x, y) \leq\|\tilde{\Phi}(x)-\tilde{\Phi}(y)\|_{\mathbb{R}^{n}} \leq \frac{\tau}{R} d_{g}(x, y)
$$

Here, the constants $\mu_{k}, k=1, \cdots, n$, satisfy

$$
\mu_{k} \leq C
$$

where $C$ is a constant dependent only on $n, Q, \kappa, \alpha, i_{0}$, and $V$.

This Theorem indicates that the mapping $\Phi$ of a local ball is an embedding and if the weighting of the parametrization is chosen property, the embedding $\tilde{\Phi}$ is with low distortion. It further shows that the eigenvalues should not be too large or too small, which means that locally the manifold could be well parameterized by "bandpass filtering".

The proof strategy of Theorem 5 is summarized below. We provide $C^{1, \alpha}$ bounds of the eigenvector fields in Proposition 8. Next, we provide estimates on $\left\|k_{T M}(t, w, z)\right\|_{H S}^{2}$ and its gradient in Lemma 10; with the bounds of eigenvector fields, we provide a control on the truncated series expansion of $\left\|k_{T M}(t, w, z)\right\|_{H S}^{2}$ and its gradient in Lemmas 11 and 12. In Lemma 13, we show how to choose desired eigenvector fields. Last, in Theorem 5. we show that the parametrization defined via those appropriately chosen eigenvector fields has the desired properties.

The key step toward the proof is the technical lemma saying that for a given eigenvector fields, locally its $C^{1, \alpha}$ norm could be well controlled by its local average $L^{2}$ norm. To obtain this technical lemma, we need the following Caccioppoli's type inequality. Note that although in this section the metric we consider is smooth, the control could be obtained when the metric is as weak as $C^{1, \alpha}$. Since the lemma has its own interest 
and we need the rough metric version for the eigenvector field perturbation argument later, we provide the proof under the weak assumption that the metric is $C^{1, \alpha}$.

Lemma 6 (Caccioppoli's type inequality). Suppose that $g \in C^{1, \alpha}$ and $U$ is a bounded solution of $\nabla^{2} U=0$ in $B=B_{R}(z)$, where $R<R_{z}$, with the Dirichlet boundary condition on $\partial B$. Then for $0<r \leq R / 2$,

$$
\|\nabla U\|_{L^{2}\left(B_{r}(z)\right)} \leq C R^{-1}\|U\|_{L^{2}(B)} .
$$

for some $C=C(Q)$.

Proof. Choose a smooth cut-off function $\psi$ on $M$ so that

$$
\begin{gathered}
0 \leq \psi \leq 1, \quad\|\nabla \psi\|_{g} \leq 2 / R \\
\psi \equiv 1 \text { on } B_{r}(z), \text { and } \\
\psi \equiv 0 \text { outside } B_{R}(z) .
\end{gathered}
$$

Since $\nabla^{2} U=0$, by integration by parts, we have

$$
\int_{B} \psi^{2}\|\nabla U\|_{g}^{2} d \sigma=\int_{B} \psi^{2} g^{a b} g_{c d} \nabla_{a} U^{c} \nabla_{b} U^{d} d \sigma=-2 \int_{B} \psi g^{a b} g_{c d} U^{d} \nabla_{a} U^{c} \nabla_{b} \psi d \sigma
$$

By the assumption that $Q^{-1} \leq|g| \leq Q$ and the choice of $\psi$, we have

$$
\int_{B} \psi^{2}\|\nabla U\|_{g}^{2} d \sigma \leq C \int_{B} \frac{2}{R} \psi\|U\|_{g}\|\nabla U\|_{g} d \sigma
$$

for some $C=C(Q)$. Applying Young's inequality and choosing $\epsilon=\frac{1}{2 C}$, we obtain

$$
\begin{aligned}
\int_{B} \psi^{2}\|\nabla U\|_{g}^{2} d \sigma & \leq \epsilon C \int_{B} \psi^{2}\|\nabla U\|_{g}^{2} d \sigma+\frac{4 C}{\epsilon R^{2}} \int_{B}\|U\|_{g}^{2} d \sigma \\
& \leq \frac{1}{2} \int_{B} \psi^{2}\|\nabla U\|_{g}^{2} d \sigma+\frac{8 C^{2}}{R^{2}} \int_{B}\|U\|_{g}^{2} d \sigma
\end{aligned}
$$

and thus

$$
\int_{B_{r}(z)}\|\nabla U\|_{g}^{2} d \sigma \leq \int_{B} \psi^{2}\|\nabla U\|_{g}^{2} d \sigma \leq \frac{16 C^{2}}{R^{2}} \int_{B}\|U\|_{g}^{2} d \sigma
$$

which implies the estimate 3.7 ).

Denote $B=B_{R}(z), R<R_{z}$. Let $\xi_{i}^{B}$ be the $i$-th eigenfunction of the LaplaceBeltrami operator $\Delta$ satisfying the Dirichlet boundary condition with the eigenvalue $-\nu_{i}^{B}$; that is, $\Delta^{B} \xi_{i}^{B}=-\nu_{i}^{B} \xi_{i}^{B}$ on $B$ and $\xi_{i}^{B}=0$ on $\partial B$. We sort the eigenvalues by $0 \leq \nu_{1}^{B} \leq \nu_{2}^{B} \leq \cdots$ and assume that the eigenfunctions $\left\{\xi_{i}^{B}\right\}$ are $L^{2}(B, g)$ normalized.

Lemma 7. Assume that $g \in C^{1, \alpha}$. Let $\xi_{k}^{B}$ be the Dirichlet eigenfunctions of $\Delta$ on $B$ with the eigenvalue $\nu_{k}^{B}$. Then we have the estimate

$$
\left\|\xi_{k}^{B} X_{i}\right\|_{L^{\frac{2 n}{n-2}}(B)} \leq C\left(\left(\nu_{k}^{B}+\lambda_{i}\right)^{1 / 2}+2 \nu_{k}^{B}\right)\left(\nu_{k}^{B}\right)^{\beta}\left\|X_{i}\right\|_{L^{2}(B)}
$$

for some $C=C(n, Q)$, where $\beta=\frac{n-1}{2}$ for $n$ odd and $\beta=\frac{n}{2}$ for $n$ even. 
Proof. By the Sobolev embedding inequality, it suffices to prove that

$$
\left\|\nabla\left(\xi_{k}^{B} X_{i}\right)\right\|_{L^{2}(B)} \leq C\left(\left(\nu_{k}^{B}+\lambda_{i}\right)^{1 / 2}+2 \nu_{k}^{B}\right)\left(\nu_{k}^{B}\right)^{\beta}\left\|X_{i}\right\|_{L^{2}(B)} .
$$

By a direct computation, we have

$$
\nabla^{2}\left(\xi_{k}^{B} X_{i}\right)=-\left(\nu_{k}^{B}+\lambda_{i}\right) \xi_{k}^{B} X_{i}+2 g^{a b} \nabla_{a} \xi_{k}^{B} \nabla_{b} X_{i}
$$

and

$$
\left\|\nabla\left(\xi_{k}^{B} X_{i}\right)\right\|_{g}^{2} \geq\left\|\xi_{k}^{B} \nabla X_{i}\right\|_{g}^{2}-2\left|g^{c d} g_{a b} \xi_{k}^{B} X_{i}^{b} \nabla_{d} \xi_{k}^{B} \nabla_{c} X_{i}^{a}\right| .
$$

Combining them, we have

$$
\begin{aligned}
\int_{B}\left\|\xi_{k}^{B} \nabla X_{i}\right\|_{g}^{2} d \sigma & \leq \int_{B}\left\|\nabla\left(\xi_{k}^{B} X_{i}\right)\right\|_{g}^{2} d \sigma+2 \int_{B}\left|g^{c d} g_{a b} \xi_{k}^{B} X_{i}^{b} \nabla_{d} \xi_{k}^{B} \nabla_{c} X_{i}^{a}\right| d \sigma \\
& =\int_{B}\left\langle\xi_{k}^{B} X_{i},-\nabla^{2}\left(\xi_{k}^{B} X_{i}\right)\right\rangle_{g} d \sigma+2 \int_{B}\left|g^{c d} g_{a b} \xi_{k}^{B} X_{i}^{b} \nabla_{d} \xi_{k}^{B} \nabla_{c} X_{i}^{a}\right| d \sigma \\
& =\int_{B}\left\langle\xi_{k}^{B} X_{i},\left(\nu_{k}^{B}+\lambda_{i}\right) \xi_{k}^{B} X_{i}+2 g^{a b} \nabla_{a} \xi_{k}^{B} \nabla_{b} X_{i}\right\rangle d \sigma \\
& \quad+2 \int_{B}\left|g^{c d} g_{a b} \xi_{k}^{B} X_{i}^{b} \nabla_{d} \xi_{k}^{B} \nabla_{c} X_{i}^{a}\right| d \sigma \\
& \leq\left(\nu_{k}^{B}+\lambda_{i}\right) \int_{B}\left\|\xi_{k}^{B} X_{i}\right\|_{g}^{2} d \sigma+4 \int_{B}\left|g^{c d} g_{a b} \xi_{k}^{B} X_{i}^{b} \nabla_{d} \xi_{k}^{B} \nabla_{c} X_{i}^{a}\right| d \sigma
\end{aligned}
$$

Applying the Cauchy-Schwarz inequality and Lemma 3.5.3 in [22], there exists some constant $C=C(n, Q)$ such that

$$
\begin{aligned}
\left\|\xi_{k}^{B} \nabla X_{i}\right\|_{L^{2}(B)}^{2} & \leq\left(\nu_{k}^{B}+\lambda_{i}\right)\left\|\xi_{k}^{B}\right\|_{L^{\infty}(B)}^{2}\left\|X_{i}\right\|_{L^{2}(B)}^{2}+4\left\|\nabla \xi_{k}^{B}\right\|_{L^{\infty}(B)}\left\|X_{i}\right\|_{L^{2}(B)}\left\|\xi_{k}^{B} \nabla X_{i}\right\|_{L^{2}(B)} \\
& \leq C\left(\nu_{k}^{B}+\lambda_{i}\right)\left(\nu_{k}^{B}\right)^{2 \beta}\left\|X_{i}\right\|_{L^{2}(B)}^{2}+4\left(\nu_{k}^{B}\right)^{\beta+1}\left\|X_{i}\right\|_{L^{2}(B)}\left\|\xi_{k}^{B} \nabla X_{i}\right\|_{L^{2}(B)}
\end{aligned}
$$

where $\beta=\frac{n-1}{2}$ for $n$ odd and $\beta=\frac{n}{2}$ for $n$ even. By a direct bound, this quadratic inequality implies that

$$
\left\|\xi_{k}^{B} \nabla X_{i}\right\|_{L^{2}(B)}^{2} \leq C\left(\left(\nu_{k}^{B}+\lambda_{i}\right)^{1 / 2}+2 \nu_{k}^{B}\right)\left(\nu_{k}^{B}\right)^{\beta}\left\|X_{i}\right\|_{L^{2}(B)}^{2}
$$

for some $C=C(n, Q)$.

Finally, we can bound $\left\|\nabla\left(\xi_{k}^{B} X_{i}\right)\right\|_{L^{2}(B)}$ by

$$
\begin{aligned}
\left\|\nabla\left(\xi_{k}^{B} X_{i}\right)\right\|_{L^{2}(B)} & \leq\left\|\nabla \xi_{k}^{B}\right\|_{L^{\infty}(B)}\left\|X_{i}\right\|_{L^{2}(B)}^{2}+\left\|\xi_{k}^{B} \nabla X_{i}\right\|_{L^{2}(B)}^{2} \\
& \leq C\left(\left(\nu_{k}^{B}+\lambda_{i}\right)^{1 / 2}+2 \nu_{k}^{B}\right)\left(\nu_{k}^{B}\right)^{\beta}\left\|X_{i}\right\|_{L^{2}(B)}^{2} .
\end{aligned}
$$

Proposition 8. Suppose $g \in C^{1, \alpha}$. Let $P_{1}(x)$ and $P_{2}(x)$ be polynomials defined as

$$
P_{1}(x)=(1+x)^{\left\lceil\frac{n-2}{4}\right\rceil} \text { and } P_{2}(x)=(1+x)^{\left\lceil\frac{n-2}{4}\right\rceil+1},
$$

where $\lceil x\rceil$ denotes the smallest integer not less than $x$.

For any $R \leq R_{z}$, we have the following for the $i$-th eigenvector field of $\nabla^{2}$ : 


$$
\begin{gathered}
\left\|X_{i}\right\|_{C^{0}\left(B_{\frac{R}{2}}(z)\right)} \leq C P_{1}\left(\lambda_{i} R^{2}\right)\left(f_{B_{R}(z)}\left\|X_{i}\right\|_{g}^{2}\right)^{1 / 2}, \\
\left\|\nabla X_{i}\right\|_{C^{0}\left(B_{\frac{R}{2}}(z)\right)} \leq C \frac{1}{R} P_{2}\left(\lambda_{i} R^{2}\right)\left(f_{B_{R}(z)}\left\|X_{i}\right\|_{g}^{2}\right)^{1 / 2}, \\
\left\|X_{i}\right\|_{C^{\alpha}\left(B_{\frac{R}{2}}(z)\right)} \leq C \frac{1}{R^{\alpha}} P_{1}\left(\lambda_{i} R^{2}\right)\left(f_{B_{R}(z)}\left\|X_{i}\right\|_{g}^{2}\right)^{1 / 2}
\end{gathered}
$$

and

$$
\left\|\nabla X_{i}\right\|_{C^{\alpha}\left(B_{\frac{R}{2}}(z)\right)} \leq C \frac{1}{R^{1+\alpha}} P_{2}\left(\lambda_{i} R^{2}\right)\left(f_{B_{R}(z)}\left\|X_{i}\right\|_{g}^{2}\right)^{1 / 2},
$$

where $C$ depends on constants $n, Q, R, \kappa$, and $\|g\|_{C^{1, \alpha}}$.

Proof. The proof relies on interior estimates [16, Lemma 4] and estimates on Green's matrix in [16. Theorem 1]. Lemma 4 is proved by using the Sobolev inequality and the Schauder's estimates, which says for harmonic vector fields $\nabla^{2} U=0$ on $B_{r}$, one has

$$
\sup _{B_{r / 2}}\|U\|_{g} \sup _{B_{r / 2}}\|\nabla U\|_{g} \leq C\left(\|\nabla U\|_{L^{2}\left(B_{r}\right)}+\|U\|_{L^{\frac{2 n}{n-2}\left(B_{r}\right)}}\right)
$$

where $C=C\left(n, Q, r, \kappa,\|g\|_{C^{1, \alpha}}\right)$.

Theorem 1 in [16] is based on the global estimates Theorems 6.4.8 and 6.5.5 in [26] and the interior estimates. In particular, the Green's matrix on $B_{r}$ has the following properties

$$
\begin{aligned}
& G \in L^{\frac{n}{n-2}, \infty}\left(B_{r}\right) \text { with }\|G\|_{L^{\frac{n}{n-2}, \infty}\left(B_{r}\right)} \leq C\left(n, Q, \kappa,\|g\|_{C^{1, \alpha}}\right) \\
& \nabla G \in L^{\frac{n}{n-1}, \infty}\left(B_{r}\right) \text { with }\|\nabla G\|_{L^{\frac{n}{n-1}, \infty}\left(B_{r}\right)} \leq C\left(n, Q, \kappa,\|g\|_{C^{1, \alpha}}\right)
\end{aligned}
$$

and

$$
\begin{aligned}
\left\|G(x, w)-P_{y}^{x} G(y, w)\right\|_{g} & \leq C \frac{d_{g}(x, y)^{\alpha}}{\max d_{g}(x, w)^{2-n-\alpha}, d_{g}(y, w)^{2-n-\alpha}} \\
\left\|D G(x, w)-P_{y}^{x} D G(y, w)\right\|_{g} & \leq C \frac{d_{g}(x, y)^{\alpha}}{\max d_{g}(x, w)^{1-n-\alpha}, d_{g}(y, w)^{1-n-\alpha}}
\end{aligned}
$$

where $C=C\left(n, Q, r, \kappa,\|g\|_{C^{1, \alpha}}\right)$.

Rescale $R$ to 1 by rescaling the eigenvector field $\tilde{X}_{i}(y)=P_{\exp _{z}\left(R \exp _{z}^{-1} y\right)}^{y} X_{i}\left(\exp _{z}\left(R \exp _{z}^{-1} y\right)\right)$, where $P_{\exp _{z}\left(R \exp _{z}^{-1} y\right)}^{y}$ is the parallel transport from $\exp _{z}\left(R \exp _{z}^{-1} y\right)$ to $y$. We have the following rescaling:

$$
\begin{gathered}
\nabla^{2} \tilde{X}_{i}(y)=\lambda_{i} R^{2} \tilde{X}_{i}(y) \\
\nabla \tilde{X}_{i}(y)=R P_{\exp _{z}\left(R \exp _{z}^{-1} y\right)}^{y} \nabla X_{i}\left(\exp _{z}\left(R \exp _{z}^{-1} y\right)\right),
\end{gathered}
$$


and by the change of variables,

$$
f_{B_{1}(z)}\left\langle\tilde{X}_{i}, \tilde{X}_{i}\right\rangle d \sigma=f_{B_{R}(z)}\left\langle X_{i}, X_{i}\right\rangle d \sigma .
$$

To simplify the notation, below we use $X_{i}$ to represent the rescaled $\tilde{X}_{i}$.

Let $r_{0}=1>r_{1}>r_{2}>\cdots>\frac{1}{2}=r_{m}$ where $m$ is to be chosen later. For $l=0,1, \ldots$, let $B_{l}=B_{r_{l}}(z)$ and let $G^{B_{l}}$ denote the Green's operator on $B_{l}$ associated with $\nabla^{2}$ with Dirichlet boundary condition. Write

$$
\left.X_{i}\right|_{B_{l}}=U_{(l)}+V_{(l)}
$$

where

$$
V_{(l)}(x)=\int_{B_{l}} G^{B_{l}}(x, y) \nabla^{2} X_{i}(y) d y=-\lambda_{i} \int_{B_{l}} G^{B_{l}}(x, y) X_{i}(y) d y
$$

and $\nabla^{2} U_{(l)}=0$ on $B_{l}$. Note that since $g \in C^{1, \alpha}, G^{B_{l}} \in L^{\frac{n}{n-2}, \infty}\left(B_{l}, T M \otimes T^{*} M\right)$ and $\nabla G^{B_{l}} \in L^{\frac{n}{n-1}, \infty}\left(B_{l}, T M \otimes T^{*} M \otimes T^{*} B\right)$ (see [16, Theorem 1]).

We start the iteration with $l=0$. Let $p_{0}=\frac{2 n}{n-2}$, which is the conjugate of 2 . Let $p_{1}=\frac{2 n}{n-6+\eta_{1}}$ for some $\eta_{1}, 0<\eta_{1}<4$. Note that $\frac{1}{p_{0}}+\frac{2 n-4+\eta_{1}}{2 n}=\frac{1}{p_{1}}+1$ and $p_{1}>p_{0}>2$. Following the proofs of Young's inequality, we have

$$
\begin{aligned}
\left\|V_{(0)}\right\|_{L^{p_{1}}\left(B_{2}\right)} & \leq\left\|V_{(0)}\right\|_{L^{p_{1}}\left(B_{0}\right)} \\
& =\lambda_{i}\left\|\int_{B_{0}} G^{B_{0}}(x, y) X_{i}(y) d y\right\|_{L^{p_{1}}\left(B_{0}\right)} \\
& \leq \lambda_{i}\left\|G^{B_{0}}\right\|_{L^{\frac{2 n}{2 n-4+\eta_{1}}\left(B_{0}\right)}}\left\|X_{i}\right\|_{L^{p_{0}}\left(B_{0}\right)}
\end{aligned}
$$

and hence

$$
\left\|V_{(0)}\right\|_{L^{p_{1}\left(B_{2}\right)}} \leq C \lambda_{i}\left\|X_{i}\right\|_{L^{p_{0}\left(B_{0}\right)}}
$$

where $C=C\left(p_{1}, p_{0}, V\right)$, since $G^{B_{0}} \in L^{\frac{n}{n-2}, \infty}$ and $\frac{2 n}{2 n-4+\eta_{1}}<\frac{n}{n-2}$. From the interior estimate in [16, Lemma 4] and the fact that $L^{p, \infty} \subset L^{p}$ for $1 \leq p<\infty$, there exists a constant $C=C\left(n, Q, r, \kappa,\|g\|_{C^{1, \alpha}}\right)$ such that

$$
\begin{aligned}
\left\|U_{(0)}\right\|_{L^{\infty}\left(B_{2}\right)} & \leq C\left(\left\|\nabla U_{(0)}\right\|_{L^{2}\left(B_{1}\right)}+\|U\|_{L^{p_{0}\left(B_{1}\right)}}\right) \\
& \leq C\left(\left\|\nabla U_{(0)}\right\|_{L^{2}\left(B_{1}\right)}+\|U\|_{L^{p_{0}\left(B_{0}\right)}}\right)
\end{aligned}
$$

from Lemma 6 and the fact that $p_{0}>2$, we have

$$
\left\|\nabla U_{(0)}\right\|_{L^{2}\left(B_{1}\right)} \leq C\left\|U_{(0)}\right\|_{L^{2}\left(B_{0}\right)} \leq C\left\|U_{(0)}\right\|_{L^{p_{0}\left(B_{0}\right)}}
$$

and hence

$$
\begin{aligned}
\left\|U_{(0)}\right\|_{L^{\infty}\left(B_{2}\right)} & \leq C\left\|U_{(0)}\right\|_{L^{p_{0}}\left(B_{0}\right)} \\
& \leq C\left(\left\|V_{(0)}\right\|_{L^{p_{0}\left(B_{0}\right)}}+\left\|X_{i}\right\|_{L^{p_{0}\left(B_{0}\right)}}\right) \\
& \leq C\left(\left\|V_{(0)}\right\|_{L^{p_{1}}\left(B_{0}\right)}+\left\|X_{i}\right\|_{L^{p_{0}\left(B_{0}\right)}}\right) \\
& \leq C\left(1+\lambda_{i}\right)\left\|X_{i}\right\|_{L^{p_{0}}\left(B_{0}\right)}
\end{aligned}
$$


where the third inequality holds since $p_{1}>p_{0}$. Eventually, we have

$$
\begin{aligned}
\left\|X_{i}\right\|_{L^{p_{1}\left(B_{2}\right)}} & \leq\left\|V_{(0)}\right\|_{L^{p_{1}\left(B_{2}\right)}}+\left\|U_{(0)}\right\|_{L^{p_{1}}\left(B_{2}\right)} \\
& \leq C \lambda_{i}\left\|X_{i}\right\|_{L^{p_{0}}\left(B_{0}\right)}+\left\|U_{(0)}\right\|_{L^{\infty}\left(B_{2}\right)} \\
& \leq C\left(1+\lambda_{i}\right)\left\|X_{i}\right\|_{L^{p_{0}}\left(B_{0}\right)} .
\end{aligned}
$$

For $l=1,2, \ldots$, let $p_{l}=\frac{2 n}{n-2-4 l+\sum_{j=1}^{l} \eta_{j}}$ where $\eta_{j}$ are constants, $0<\eta_{j}<4$ for all $j=1, \cdots, l$. Based on the same argument, by the fact that $B_{l+1} \subset B_{l}$ and $\frac{1}{p_{l}}+\frac{2 n-4+\eta_{l+1}}{2 n}=\frac{1}{p_{l+1}}+1$, we have

$$
\begin{aligned}
\left\|V_{(l)}\right\|_{L^{p_{l+1}\left(B_{l+2}\right)}} & \leq\left\|V_{(l)}\right\|_{L^{p_{l+1}\left(B_{l}\right)}} \\
& \leq \lambda_{i}\left\|G^{B_{l}}\right\|_{L^{2 n-4+\eta_{l+1}}\left(B_{l}\right)}\left\|X_{i}\right\|_{L^{p_{l}}\left(B_{l}\right)} \\
& \leq C \lambda_{i}\left\|X_{i}\right\|_{L^{p_{l}\left(B_{l}\right)} .}
\end{aligned}
$$

Similarly, from the interior estimate in [16, Lemma 4] and Lemma 6, there exists a constant $C=C\left(n, Q, r, \kappa,\|g\|_{C^{1, \alpha}}\right)$ such that

$$
\left\|U_{(l)}\right\|_{L^{\infty}\left(B_{l+2}\right)} \leq C\left\|U_{(l)}\right\|_{L^{p_{l}\left(B_{l}\right)}} \leq C\left(1+\lambda_{i}\right)\left\|X_{i}\right\|_{L^{p_{l}\left(B_{l}\right)}}
$$

and hence

$$
\begin{aligned}
\left\|X_{i}\right\|_{L^{p^{l+1}\left(B_{l+1}\right)}} & \leq\left\|V_{(l)}\right\|_{L^{p_{l+1}\left(B_{l+1}\right)}}+\left\|U_{(l)}\right\|_{L^{p_{l+1}\left(B_{l+1}\right)}} \\
& \leq C \lambda_{i}\left\|X_{i}\right\|_{L^{p_{l}\left(B_{l}\right)}}+\left\|U_{(l)}\right\|_{L^{\infty}\left(B_{l+1}\right)} \\
& \leq C\left(1+\lambda_{i}\right)\left\|X_{i}\right\|_{L^{p_{l}\left(B_{l}\right)}} .
\end{aligned}
$$

By the iteration, we have

$$
\left\|X_{i}\right\|_{L^{p_{l+1}\left(B_{l+2}\right)}} \leq C\left(1+\lambda_{i}\right)^{l+1}\left\|X_{i}\right\|_{L^{p_{0}}\left(B_{0}\right)} .
$$

Let $m$ to be the smallest integer greater or equal to $\frac{n-2}{2}$. We may choose constants $\eta_{1}, \cdots, \eta_{m}$ so that $p_{m}=\infty$. Then we have

$$
\left\|X_{i}\right\|_{L^{\infty}\left(B_{r_{m}}(z)\right)} \leq C\left(1+\lambda_{i}\right)^{m}\left\|X_{i}\right\|_{L^{p_{0}(B)}} .
$$

To control $\left\|X_{i}\right\|_{L^{p_{0}(B)}}$, we let $\psi=\sum_{i=1}^{K} a_{i} \xi_{j}^{B}$ be a finite sum of Dirichlet eigenfunctions on $B$ such that

$$
\frac{1}{2} \leq \psi(x) \leq 2, \text { for } x \in B
$$


and $\sum_{i=1}^{K}\left|a_{i}\right| \leq C, \nu_{i}^{B} \leq C$, for all $1 \leq i \leq K$. By Lemma 7 ,

$$
\begin{aligned}
\left\|X_{i}\right\|_{L^{p_{0}}(B)} & \leq 2\left\|\psi X_{i}\right\|_{L^{\frac{2 n}{n-2}}(B)} \\
& \leq 2 \sum_{k=1}^{K}\left|a_{k}\right|\left\|\xi_{k}^{B} X_{i}\right\|_{L^{\frac{2 n}{n-2}}(B)} \\
& \leq 2 \sum_{k=1}^{K}\left|a_{k}\right|\left(\left(\nu_{k}^{B}+\lambda_{i}\right)^{1 / 2}+2 \nu_{k}^{B}\right)\left(\nu_{k}^{B}\right)^{\beta}\left\|X_{i}\right\|_{L^{2}(B)} \\
& \leq C\left(\lambda_{i}+1\right)^{1 / 2}\left\|X_{i}\right\|_{L^{2}(B)}
\end{aligned}
$$

which, when combined with (3.46), implies the estimate (3.22).

To bound $\left\|\nabla X_{i}\right\|_{L^{\infty}\left(B_{\frac{1}{2}}(z)\right)}=\left\|\nabla X_{i}\right\|_{L^{\infty}\left(B_{m}\right)}$, we follow the same line by noting that

$$
\begin{aligned}
\left\|\nabla V_{(m-1)}\right\|_{L^{\infty}\left(B_{m}\right)} & =\left\|\nabla \int G^{B_{1 / 2}(z)}(x, y) \nabla^{2} X_{i}(y) d \sigma\right\|_{L^{\infty}\left(B_{\frac{1}{2}}(z)\right)} \\
& \leq \lambda_{i}\left\|\nabla G^{B_{1 / 2}}\right\|_{L^{1}\left(B_{1 / 2}\right)}\left\|X_{i}\right\|_{L^{\infty}\left(B_{\frac{1}{2}}(z)\right)} \\
& \leq C \lambda_{i}\left(1+\lambda_{i}\right)^{m}\left\|X_{i}\right\|_{L^{2}(B)} .
\end{aligned}
$$

Again by the interior estimate in [16, Lemma 4] and Lemma 6, there exists a constant $C=C\left(n, Q, r, \kappa,\|g\|_{C^{1, \alpha}}\right)$ such that

$$
\left\|\nabla U_{(m-1)}\right\|_{L^{\infty}\left(B_{m}\right)} \leq C\left\|U_{(m-1)}\right\|_{L^{p_{0}\left(B_{m-1}\right)}} .
$$

The bound $(3.23)$ follows from $(3.49)$ and $(3.50)$.

Finally, to control the Hölder seminorm in (3.24) and (3.25), we need we first choose a cut-off function $\eta$ so that $0 \leq \eta \leq 1$, and

$$
\begin{gathered}
\eta=1 \text { on } B_{1 / 2}(z) \\
\eta=0 \text { outside of } B_{3 / 4}(z) \\
|\nabla \eta|,|\Delta \eta| \leq 4 .
\end{gathered}
$$

Jones-Maggioni-Schul provided an example of such a cut-off function in [22, p. 162].

Let $B_{r}=B_{r}(z)$ and $B=B_{1}$. Let $G$ be the Green's operator on $B_{3 / 4}$ with the Dirichlet boundary condition. Then

$$
\begin{aligned}
\left|X_{i}(x)-P_{y}^{x} X_{i}(y)\right| & \leq \int_{B_{3 / 4}(z)}\left(G(x, w)-P_{y}^{x} G(y, w)\right)\left(\nabla^{2}\left(\eta X_{i}\right)\right)(w) d \sigma(w) \\
& \leq\left\|\nabla^{2}\left(\eta X_{i}\right)\right\|_{L^{\infty}(B)} \int_{B}|(G(x, w)-G(y, w))| d \sigma(w)
\end{aligned}
$$

Note that

$$
\begin{aligned}
& \left\|\nabla^{2}\left(\eta X_{i}\right)\right\|_{L^{\infty}\left(B_{3 / 4}\right)} \\
& \leq\left\|\eta \nabla^{2} X_{i}\right\|_{L^{\infty}\left(B_{3 / 4}\right)}+\left\|(\Delta \eta) X_{i}\right\|_{L^{\infty}\left(B_{3 / 4}\right)}+2\|\nabla \eta\|_{L^{\infty}\left(B_{3 / 4}\right)}\left\|\nabla X_{i}\right\|_{L^{\infty}\left(B_{3 / 4}\right)} \\
& \leq\left(\|\Delta \eta\|_{L^{\infty}(B)}+\lambda_{i}\|\eta\|_{L^{\infty}(B)}\right)\left\|X_{i}\right\|_{L^{\infty}(B)}+\|\nabla \eta\|_{L^{\infty}(B)}\left\|\nabla X_{i}\right\|_{L^{\infty}(B)} \\
& \leq 4\left(\left(1+\lambda_{i}\right) P_{1}\left(\lambda_{i}\right)+P_{2}\left(\lambda_{i}\right)\right)\left\|X_{i}\right\|_{L^{2}(B)}
\end{aligned}
$$


where the last inequality follows from the bounds $(3.22)$ and $(3.23)$.

On the other hand, by the pointwise estimates of the Green't matrix in Theorem 1 in [16], we have, for $|v|=k$,

$$
\left|\nabla^{v} G(x, w)-P_{y}^{x} \nabla^{v} G(y, w)\right| \leq C \frac{d_{g}(x, y)^{\alpha}}{\max \left\{d_{g}(x, w)^{2-n-k-\alpha}, d_{g}(y, w)^{2-n-k-\alpha}\right\}} .
$$

Combining (3.51), 3.52), and (3.53), we have

$$
\left|X_{i}(x)-P_{y}^{x} X_{i}(y)\right| \leq C P_{2}\left(\lambda_{i}\right) d_{g}(x, y)^{\alpha}\left\|X_{i}\right\|_{L^{2}(B)}
$$

which implies (3.24) after rescaling 1 back to $R$.

Similary, since

$$
\begin{aligned}
\left|\nabla X_{i}(x)-P_{y}^{x} \nabla X_{i}(y)\right| & \leq \int_{B_{3 / 4}(z)}\left(\nabla G(x, w)-P_{y}^{x} \nabla G(y, w)\right)\left(\nabla^{2}\left(\eta X_{i}\right)\right)(w) d \sigma(w) \\
& \leq\left\|\nabla^{2}\left(\eta X_{i}\right)\right\|_{L^{\infty}(B)} \int_{B}|(G(x, w)-G(y, w))| d \sigma(w)
\end{aligned}
$$

the bound (3.25) follows from (3.55), 3.52), and (3.53).

Corollary 9. Let $\alpha, P_{1}(x)$ and $P_{2}(x)$ be defined as in Proposition 8. For $R \leq R_{z}$, $x, y \in B_{\frac{R}{2}}(z)$, there exists a constant $C=C\left(n, Q, r, \kappa,\|g\|_{C^{1, \alpha}}\right)$ such that the following estimates hold

$$
\left|\left\langle X_{i}, X_{j}\right\rangle(x)\right| \leq C P_{1}\left(\lambda_{i} R^{2}\right) P_{1}\left(\lambda_{j} R^{2}\right)\left(f_{B_{R}(z)}\left\|X_{i}\right\|_{g}^{2}\right)^{1 / 2}\left(f_{B_{R}(z)}\left\|X_{j}\right\|_{g}^{2}\right)^{1 / 2}
$$

$$
\left\|\nabla\left\langle X_{i}, X_{j}\right\rangle(x)\right\|_{g} \leq C \frac{1}{R} P_{2}\left(\lambda_{i} R^{2}\right) P_{2}\left(\lambda_{j} R^{2}\right)\left(f_{B_{R}(z)}\left\|X_{i}\right\|_{g}^{2}\right)^{1 / 2}\left(f_{B_{R}(z)}\left\|X_{j}\right\|_{g}^{2}\right)^{1 / 2},
$$

and

$$
\begin{aligned}
& \left\|\nabla\left\langle X_{i}, X_{j}\right\rangle(x)-P_{y}^{x} \nabla\left\langle X_{i}, X_{j}\right\rangle(y)\right\|_{g} \\
\leq & C \frac{d_{g}(x, y)^{\alpha}}{R^{1+\alpha}} P_{2}\left(\lambda_{i} R^{2}\right) P_{2}\left(\lambda_{j} R^{2}\right)\left(f_{B_{R}(z)}\left\|X_{i}\right\|_{g}^{2}\right)^{1 / 2}\left(f_{B_{R}(z)}\left\|X_{j}\right\|_{g}^{2}\right)^{1 / 2} .
\end{aligned}
$$

Proof. The inequality (3.56) follows by the Cauchy-Schwarz inequality and (3.22) in Proposition 8;

$$
\begin{aligned}
\left|\left\langle X_{i}, X_{j}\right\rangle(x)\right| & \leq\left\|X_{i}\right\|_{C^{0}\left(B_{\frac{R}{2}}(z)\right)}\left\|X_{j}\right\|_{C^{0}\left(B_{\frac{R}{2}}(z)\right)} \\
& \leq C P_{1}\left(\lambda_{i} R^{2}\right) P_{1}\left(\lambda_{j} R^{2}\right)\left(f_{B_{R}(z)}\left\|X_{i}\right\|_{g}^{2}\right)^{1 / 2}\left(f_{B_{R}(z)}\left\|X_{j}\right\|_{g}^{2}\right)^{1 / 2} .
\end{aligned}
$$

Similarly, using (3.22), 3.23), and the Cauchy-Schwarz inequality, we have

$$
\begin{aligned}
\left\|\nabla\left\langle X_{i}, X_{j}\right\rangle(x)\right\|_{g} & \leq\left\|\nabla X_{i}\right\|_{C^{0}\left(B_{\frac{R}{2}}(z)\right)}\left\|X_{j}\right\|_{C^{0}\left(B_{\frac{R}{2}}(z)\right)}+\left\|\nabla X_{j}\right\|_{C^{0}\left(B_{\frac{R}{2}}(z)\right)}\left\|X_{i}\right\|_{C^{0}\left(B_{\frac{R}{2}}(z)\right)} \\
& \leq C \frac{1}{R} P_{2}\left(\lambda_{i} R^{2}\right) P_{2}\left(\lambda_{j} R^{2}\right)\left(f_{B_{R}(z)}\left\|X_{i}\right\|_{g}^{2}\right)^{1 / 2}\left(f_{B_{R}(z)}\left\|X_{j}\right\|_{g}^{2}\right)^{1 / 2},
\end{aligned}
$$


which gives (3.57). Last, we prove the estimate (3.58). Note that since

$$
\begin{aligned}
& \left\langle\nabla X_{i}, X_{j}\right\rangle(x)-\left\langle\nabla X_{i}, X_{j}\right\rangle(y) \\
= & \left\langle\nabla X_{i}(x), X_{j}(x)-P_{y}^{x} X_{j}(y)\right\rangle+\left\langle P_{x}^{y} \nabla X_{i}(x)-\nabla X_{i}(y), X_{j}(y)\right\rangle,
\end{aligned}
$$

we have

$$
\begin{aligned}
& \left\|\nabla\left\langle X_{i}, X_{j}\right\rangle(x)-\nabla\left\langle X_{i}, X_{j}\right\rangle(y)\right\|_{g} \\
& \leq\left\|\nabla X_{i}\right\|_{g}(x)\left\|X_{j}(x)-P_{y}^{x} X_{j}(y)\right\|_{g}+\left\|X_{j}\right\|_{g}(y)\left\|\nabla X_{i}(x)-P_{y}^{x} \nabla X_{i}(y)\right\|_{g} \\
& \quad+\left\|X_{i}\right\|_{g}(x)\left\|\nabla X_{j}(x)-P_{y}^{x} \nabla X_{j}(y)\right\|_{g}+\left\|\nabla X_{j}\right\|_{g}(y)\left\|X_{i}(x)-P_{y}^{x} X_{i}(y)\right\|_{g} .
\end{aligned}
$$

Then (3.58) follows by Proposition 8 that

$$
\begin{aligned}
& \left\|\nabla\left\langle X_{i}, X_{j}\right\rangle(x)-\nabla\left\langle X_{i}, X_{j}\right\rangle(y)\right\|_{g} \\
\leq & C \frac{d_{g}(x, y)^{\alpha}}{R^{1+\alpha}} P_{2}\left(\lambda_{i} R^{2}\right) P_{2}\left(\lambda_{j} R^{2}\right)\left(f_{B_{R}(z)}\left\|X_{i}\right\|_{g}^{2}\right)^{1 / 2}\left(f_{B_{R}(z)}\left\|X_{j}\right\|_{g}^{2}\right)^{1 / 2} .
\end{aligned}
$$

Next, we consider the heat kernel truncation approximation. Let

$$
\Lambda_{L}(A)=\left\{i: \lambda_{i} \leq A t^{-1}\right\} \text { and } \Lambda_{H}\left(A^{\prime}\right)=\left\{i: \lambda_{i}>A^{\prime} t^{-1}\right\}
$$

where $A, A^{\prime}$ are chosen positive numbers. Intuitively, $\Lambda_{L}(A)$ includes all "low frequency" eigenvector fields while $\Lambda_{H}\left(A^{\prime}\right)$ includes all "high frequency" eigenvector fields.

Lemma 10. Let $M$ be a smooth closed manifold with a smooth metric $g$. Under Assumption 4, we have the following expansions:

$$
\left\|k_{T M}(t, w, z)\right\|_{H S}^{2}=\frac{1}{(4 \pi t)^{n}}(n+O(t))\left(1-\frac{d_{g}(z, w)}{2 t}+O\left(\frac{d_{g}(z, w)^{4}}{t^{2}}\right)\right) .
$$

For $v \in T_{w} M$ a unit vector parallel to $\exp _{w}^{-1}(z)$,

$$
\left|\nabla_{v}\left\|k_{T M}(t, w, z)\right\|_{H S}^{2}\right|=\frac{1}{(4 \pi t)^{n}}(n+O(t))\left(\frac{d_{g}(z, w)}{2 t}+O\left(\frac{d_{g}(z, w)^{3}}{t^{2}}\right)\right) .
$$

Proof. By the assumption, $d_{g}(z, w)^{2} \ll t$. The estimate 3.65 and 3.66 follow from the asymptotic expansion (see [33, p. 1094] and [8, p. 87])

$$
\left\|k_{T M}(t, w, z)\right\|_{H S}^{2}=(n+O(t))(4 \pi t)^{-n}\left(1-\frac{d_{g}(z, w)^{2}}{2 t}+O\left(\frac{d_{g}(z, w)^{4}}{t^{2}}\right)\right)
$$

and its gradient follows from straightforward computation

$$
\left|\nabla_{v}\left\|k_{T M}(t, w, z)\right\|_{H S}^{2}\right|=(n+O(t))(4 \pi t)^{-n}\left(\frac{d_{g}(z, w)}{2 t}+O\left(\frac{d_{g}(z, w)^{3}}{t^{2}}\right)\right)
$$

which gives estimate 3.66 . 
Lemma 11. Under Assumption 4, for sufficiently large $A=A\left(n, Q, \kappa, \delta_{0}, \delta_{1}\right)>1$ and sufficiently small $A^{\prime}=A^{\prime}\left(n, Q, \kappa,\|g\|_{C^{1, \alpha}}, \delta_{1}\right)<1$, we can control the tail of the heat kernel. More precisely, there exist constants $c=c\left(A, A^{\prime}, n, Q, \kappa,\|g\|_{C^{1, \alpha}}, \delta_{0}, \delta_{1}\right)$ and $C=C\left(A, A^{\prime}, n, Q, \kappa,\|g\|_{C^{1, \alpha}}, \delta_{0}, \delta_{1}\right)$ so that

$$
\left\|k_{T M}(t, w, z)\right\|_{H S}^{2} \sim_{c}^{C} \sum_{i, j \in \Lambda_{L}(A)} e^{-\left(\lambda_{i}+\lambda_{j}\right) t}\left\langle X_{i}(z), X_{j}(z)\right\rangle\left\langle X_{i}(w), X_{j}(w)\right\rangle,
$$

and

$$
\|\nabla\| k_{T M}(t, w, z)\left\|_{H S}^{2}\right\|_{g} \sim_{c}^{C}\left\|\sum_{i, j \in \Lambda_{L}(A) \cap L_{H}\left(A^{\prime}\right)} e^{-\left(\lambda_{i}+\lambda_{j}\right) t}\left\langle X_{i}(w), X_{j}(w)\right\rangle \nabla\left\langle X_{i}(z), X_{j}(z)\right\rangle\right\|_{g},
$$

where $c \rightarrow 1$ as $A^{\prime} \rightarrow 0$ and $C \rightarrow 1$ as $A \rightarrow \infty$.

Proof. First, note that by the Cauchy-Schwartz inequality, we have

$$
\left\|k_{T M}(t, w, z)\right\|_{H S}^{2} \leq\left\|k_{T M}(t, z, z)\right\|_{H S}\left\|k_{T M}(t, w, w)\right\|_{H S}
$$

and hence we can bound the tail of the series by

$$
\begin{aligned}
& \left|\sum_{\lambda_{i}>A t^{-1}} \sum_{\text {or } \lambda_{j}>A t^{-1}} e^{-\left(\lambda_{i}+\lambda_{j}\right) t}\left\langle X_{i}(z), X_{j}(z)\right\rangle\left\langle X_{i}(w), X_{j}(w)\right\rangle\right| \\
& \leq e^{-A} \sum_{\lambda_{i}>A t^{-1}} e^{-\left(\lambda_{i}+\lambda_{j}\right) \frac{t}{2}}\left|\left\langle X_{i}(z), X_{j}(z)\right\rangle\left\langle X_{i}(w), X_{j}(w)\right\rangle\right| \\
& \leq e^{-A}\left\|k_{T M}\left(\frac{t}{2}, z, z\right)\right\|_{H S}\left\|k_{T M}\left(\frac{t}{2}, w, w\right)\right\|_{H S} \\
& \leq e^{-A} k_{M}\left(\frac{t}{2}, z, z\right) k_{M}\left(\frac{t}{2}, w, w\right)
\end{aligned}
$$

where the last inequality holds since $\left\|k_{T M}(t, x, x)\right\|_{H S}^{2} \leq k_{M}(t, x, x)$ for all $t>0$ and $x \in M$ [6, p.137]. Recall the upper bound the the heat kernel estimate under our manifold assumption [24, Corollary 3.1]

$$
k_{M}(t, x, y) \leq \frac{C(\epsilon)^{\alpha}}{\sqrt{\left|B_{\sqrt{t}}(x)\right|\left|B_{\sqrt{t}}(y)\right|}} \exp \left\{-\frac{d_{g}^{2}(x, y)}{(4+\epsilon) t}+\frac{C(n) \epsilon \kappa t}{\alpha-1}\right\}
$$

for any $1<\alpha<2,0<\epsilon<1$ and $C(\epsilon) \rightarrow \infty$ as $\epsilon \rightarrow 0$. By taking $\epsilon=1 / 2$ and $\alpha=3 / 2$, we clearly have that

$$
k_{M}(t, x, x) \leq C t^{-n / 2},
$$

where $C=C(n, Q, \kappa)$. Thus, we have

$$
\mid \sum_{\lambda_{i}>A t^{-1}} \text { or }{ }_{\lambda_{j}>A t^{-1}} e^{-\left(\lambda_{i}+\lambda_{j}\right) t}\left\langle X_{i}(z), X_{j}(z)\right\rangle\left\langle X_{i}(w), X_{j}(w)\right\rangle \mid \leq C(n, Q, \kappa) e^{-A} t^{-n}
$$

which implies (3.67) by choosing $A$ large enough. 
For the gradient, note that

$$
\nabla\left\|k_{T M}(t, w, z)\right\|_{H S}^{2}=\sum_{i, j} e^{-\left(\lambda_{i}+\lambda_{j}\right) t}\left\langle X_{i}(w), X_{j}(w)\right\rangle \nabla\left\langle X_{i}(z), X_{j}(z)\right\rangle .
$$

We first consider the contribution of the high frequency part; that is, when $\lambda_{i}$ or $\lambda_{j}$ is large enough. By a direct bound, we have

$$
\begin{aligned}
& \| \sum_{\lambda_{i}>A t^{-1}} \text { or } \lambda_{j}>A t^{-1} \\
& \leq 2 \sum_{\left\{i, j: \lambda_{i}>A t^{-1}\right\}} e^{-\left(\lambda_{i}+\lambda_{j}\right) t}\left\langle X_{i}(w), X_{j}(w)\right\rangle \nabla\left\langle X_{i}(z), X_{j}(z)\right\rangle \|_{g}
\end{aligned}
$$

Since $\lambda_{i} \geq A t^{-1}$ and $\lambda_{j} \geq \lambda_{j} / 2$, we have

$$
\begin{gathered}
\\
\left\|\sum_{\lambda_{i}>A t^{-1}} \sum_{\text {Or } \lambda_{j}>A t^{-1}} e^{-\left(\lambda_{i}+\lambda_{j}\right) t}\left\langle X_{i}(w), X_{j}(w)\right\rangle \nabla\left\langle X_{i}(z), X_{j}(z)\right\rangle\right\|_{g} \\
\leq 2 e^{-\frac{A}{2}} \sum_{\left\{i, j: \lambda_{i}>A t^{-1}\right\}} e^{-\left(\lambda_{i}+\lambda_{j}\right) \frac{t}{2}}\left|\left\langle X_{i}(w), X_{j}(w)\right\rangle\right|\left\|\nabla\left\langle X_{i}(z), X_{j}(z)\right\rangle\right\|_{g} \\
\left.\leq 2 e^{-\frac{A}{2}}\left(\sum_{\left\{i, j: \lambda_{i}>A t^{-1}\right\}} e^{-\left(\lambda_{i}+\lambda_{j}\right) \frac{t}{2}}\left\langle X_{i}(w), X_{j}(w)\right\rangle^{2}\right)^{1 / 2}\right)^{1 / 2} \\
\quad \times\left(\sum_{\left\{i, j: \lambda_{i}>A t^{-1}\right\}} e^{-\left(\lambda_{i}+\lambda_{j}\right) \frac{t}{2}}\left\|\nabla\left\langle X_{i}(z), X_{j}(z)\right\rangle\right\|_{g}^{2}\right)^{\prime}
\end{gathered}
$$

where the last inequality follows from the Cauchy-Schwartz inequality.

To control $\sum_{\left\{i, j: \lambda_{i}>A t^{-1}\right\}} e^{-\left(\lambda_{i}+\lambda_{j}\right) \frac{t}{2}}\left\|\nabla\left\langle X_{i}(z), X_{j}(z)\right\rangle\right\|_{g}^{2}$, we need the following bounds (3.76), 3.77), and (3.78). By (3.57) in Corollary 9, we have

$$
\begin{aligned}
& e^{-\left(\lambda_{i}+\lambda_{j}\right) \frac{t}{2}}\left\|\nabla\left\langle X_{i}(z), X_{j}(z)\right\rangle\right\|_{g}^{2} \\
\leq & e^{-\left(\lambda_{i}+\lambda_{j}\right) \frac{t}{2}} \frac{C}{R^{2}} P_{2}\left(\lambda_{i} R^{2}\right)^{2} P_{2}\left(\lambda_{j} R^{2}\right)^{2} f_{B_{R}(z)}\left\|X_{i}\right\|_{g}^{2} f_{B_{R}(z)}\left\|X_{j}\right\|_{g}^{2},
\end{aligned}
$$

where $C=C\left(n, Q, \kappa,\|g\|_{C^{1, \alpha}}\right)$. Since $e^{-\left(\lambda_{i}+\lambda_{j}\right) \frac{t}{4}}$ decays exponentially as $i$ increases and $P_{2}\left(\lambda_{i} R^{2}\right)$ increases polynomially, by the choice of $A$ and $\delta_{0}$ so that $\delta_{0}^{2} R^{2} / 4<t$, we have

$$
e^{-\left(\lambda_{i}+\lambda_{j}\right) \frac{t}{4}} P_{2}\left(\lambda_{i} R^{2}\right)^{2} P_{2}\left(\lambda_{j} R^{2}\right)^{2} \leq e^{-\left(\lambda_{i}+\lambda_{j}\right) \frac{t}{4}} P_{2}\left(\lambda_{i} \frac{4 t}{\delta_{0}^{2}}\right)^{2} P_{2}\left(\lambda_{j} \frac{4 t}{\delta_{0}^{2}}\right)^{2}
$$

which is bounded by a constant depending on $t$ and $\delta_{0}$. 
Furthermore, by the Cauchy-Schwarz inequality and Kato's inequality, we have

$$
\begin{aligned}
& \sum_{i, j} e^{-\left(\lambda_{i}+\lambda_{j}\right) \frac{t}{4}}\left\|X_{i}(x)\right\|_{g}^{2}\left\|X_{j}(y)\right\|_{g}^{2} \\
= & \left(\sum_{i} e^{-\frac{\lambda_{i} t}{4}}\left\|X_{i}(x)\right\|_{g}^{2}\right)\left(\sum_{j} e^{-\frac{\lambda_{j} t}{4}}\left\|X_{j}(y)\right\|_{g}^{2}\right) \\
\leq & \left(\sum_{i} e^{-\frac{\lambda_{i} t}{4}}\right)\left(\sum_{i} e^{-\frac{\lambda_{i} t}{4}}\left\|X_{i}(x)\right\|_{g}^{4}\right)^{1 / 2}\left(\sum_{j} e^{-\frac{\lambda_{j} t}{4}}\left\|X_{j}(y)\right\|_{g}^{4}\right)^{1 / 2} \\
\leq & n\left(\int_{M} k_{M}\left(\frac{t}{4}, x, x\right) d \sigma\right) k_{M}\left(\frac{t}{8}, x, x\right) k_{M}\left(\frac{t}{8}, y, y\right),
\end{aligned}
$$

where $k_{M}(t, \cdot, \cdot)$ denotes the heat kernel of the Laplace-Beltrami operator. To be more precise, in (3.78), we apply

$$
\sum_{i} e^{-\frac{\lambda_{i} t}{4}} \leq n \sum_{i} e^{-\frac{\nu_{i} t}{4}}=n \int_{M} k_{M}\left(\frac{t}{4}, x, x\right) d \sigma
$$

by Kato's inequality, where $\nu_{i}$ are eigenvalues of the Laplace-Beltrami operator, and

$$
\begin{aligned}
\sum_{i} e^{-\frac{\lambda_{i} t}{4}}\left\|X_{i}(x)\right\|_{g}^{4} & \leq \sum_{i, j} e^{-\frac{\left(\lambda_{i}+\lambda_{j}\right) t}{8}}\left\langle X_{i}(x), X_{j}(x)\right\rangle^{2} \\
& =\left\|k_{T M}\left(\frac{t}{8}, x, x\right)\right\|_{H S}^{2} \leq k_{M}\left(\frac{t}{8}, x, x\right),
\end{aligned}
$$

where the first inequality holds since $\left\langle X_{i}(x), X_{j}(x)\right\rangle^{2} \geq 0$ for all $i, j$ and the last inequality holds, again, due to the fact that $\left\|k_{T M}(t, x, x)\right\|_{H S}^{2} \leq k_{M}(t, x, x)$ for all $t>0$ and $x \in M$ (see [6, p.137].)

Using the bounds 3.75, 3.76), 3.77), 3.78) and (3.72), we can bound the contribution of the high frequency part

$$
\begin{aligned}
& \| \sum_{\lambda_{i}>A t^{-1}} \text { or } \lambda_{j}>A t^{-1} \\
& e^{-\left(\lambda_{i}+\lambda_{j}\right) t}\left\langle X_{i}, X_{j}\right\rangle(w) \nabla\left\langle X_{i}, X_{j}\right\rangle(z) \|_{g} \\
& \leq \frac{C}{R} t^{-\frac{3 n}{4}} e^{-\frac{A}{2}}\left(f_{B_{R}(z)} k_{M}\left(\frac{t}{8}, x, x\right) d \sigma f_{B_{R}(z)} k_{M}\left(\frac{t}{8}, y, y\right) d \sigma\right)^{1 / 2} \\
& \leq \frac{C}{R} e^{-\frac{A}{2}} t^{-\frac{5 n}{4}} \leq \frac{C}{\delta_{0}^{5 / 2} R^{7 / 2}} e^{-\frac{A}{2}}
\end{aligned}
$$

where the last inequality holds due to the choice of $\delta_{0} R_{z} \ll t^{1 / 2}$, which is arbitrarily small provided $A$ is sufficiently large. 
To bound the contribution of the low frequency part for the gradient, we proceed as below:

$$
\begin{aligned}
& \left\|\sum_{i \notin \Lambda_{H}\left(A^{\prime}\right) \text { or } j \notin \Lambda_{H}\left(A^{\prime}\right)} e^{-\left(\lambda_{i}+\lambda_{j}\right) t}\left\langle X_{i}(w), X_{j}(w)\right\rangle \nabla\left\langle X_{i}(z), X_{j}(z)\right\rangle\right\|_{g} \\
& \leq 2 \sum_{i \notin \Lambda_{H}\left(A^{\prime}\right), j} e^{-\left(\lambda_{i}+\lambda_{j}\right) t}\left|\left\langle X_{i}(w), X_{j}(w)\right\rangle\right|\left\|\nabla\left\langle X_{i}(z), X_{j}(z)\right\rangle\right\|_{g} \\
& \leq 2\left\|k_{T M}(t, w, w)\right\|_{H S}\left(\sum_{i \notin \Lambda_{H}\left(A^{\prime}\right), j} e^{-\left(\lambda_{i}+\lambda_{j}\right) t}\left\|\nabla\left\langle X_{i}(z), X_{j}(z)\right\rangle\right\|_{g}^{2}\right)^{1 / 2}
\end{aligned}
$$

where the last inequality holds by the Cauchy-Schwartz inequality. We can further bound the last term by the following:

$$
\begin{aligned}
& \sum_{i \notin \Lambda_{H}\left(A^{\prime}\right), j} e^{-\left(\lambda_{i}+\lambda_{j}\right) t}\left\|\nabla\left\langle X_{i}(z), X_{j}(z)\right\rangle\right\|_{g}^{2} \\
\leq & C \sum_{i \notin \Lambda_{H}\left(A^{\prime}\right), j} e^{-\left(\lambda_{i}+\lambda_{j}\right) t} \frac{P_{2}\left(\lambda_{i} \frac{4 t}{\delta_{1}^{2}}\right)^{2} P_{2}\left(\lambda_{j} \frac{4 t}{\delta_{1}^{2}}\right)^{2}}{R^{2}} f_{B_{R}(z)}\left\|X_{i}\right\|_{g}^{2} f_{B_{R}(z)}\left\|X_{j}\right\|_{g}^{2} \\
\leq & \frac{C}{R^{2}} P_{2}\left(\frac{4 A^{\prime}}{\delta_{1}^{2}}\right)\left(\sum_{i \notin \Lambda_{H}\left(A^{\prime}\right)} e^{-\lambda_{i} t} f_{B_{R}(z)}\left\|X_{i}\right\|_{g}^{2}\right)\left(\sum_{j} e^{-\lambda_{j} t} P_{2}\left(\lambda_{j} \frac{4 t}{\delta_{1}^{2}}\right)^{2} f_{B_{R}(z)}\left\|X_{j}\right\|_{g}^{2}\right) \\
\leq & \frac{C}{R^{2}}\left(\int_{M} k_{M}\left(\frac{t}{2}, x, x\right)\right)^{1 / 2}\left(f_{B_{R}(z)}\left\|k_{T M}\left(\frac{t}{4}, x, x\right)\right\|_{H S}\right)^{2}\left(\sum_{\lambda_{i}<A^{\prime} t^{-1}} e^{-\lambda_{i} t}\right)^{1 / 2},
\end{aligned}
$$

where the first inequality holds due to $(3.57)$ in Corollary 9 and the last inequality holds by similar arguments as in (3.76), (3.77), and (3.78).

By Weyl's law for the connection Laplacian (see [8, p.92] or [44, Lemma 4.2]) and the Kato's type inequality (see [6, p.135]),

$$
\sum_{\lambda_{i} \leq A^{\prime} t^{-1}} e^{-\lambda_{i} t} \leq e \sum_{\lambda_{i} \leq A^{\prime} t^{-1}} e^{-\frac{\lambda_{i} t}{A^{\prime}}} \leq e n \int_{M} k_{M}\left(\frac{t}{A^{\prime}}, x, x\right) d \sigma \leq e n\left(A^{\prime} t^{-1}\right)^{n / 2}
$$

where the last inequality follows from Proposition 3.1.2 in [22]. Therefore,

$$
\begin{aligned}
& \left\|\sum_{\lambda_{i}<A^{\prime} t^{-1} \text { Or } \lambda_{j}<A^{\prime} t^{-1}} e^{-\left(\lambda_{i}+\lambda_{j}\right) t}\left\langle X_{i}, X_{j}\right\rangle(w) \nabla\left\langle X_{i}, X_{j}\right\rangle(z)\right\|_{g} \\
\leq & \frac{C}{R} t^{-3 n / 2} A^{\prime \frac{n}{4}} \leq \frac{C}{\delta_{0}^{3 n} R^{3 n+1}} A^{\prime \frac{n}{4}},
\end{aligned}
$$

which implies 3.68 if $A^{\prime}$ is sufficiently small.

To finish the heat kernel truncation approximation, we further restrict ourselves on the subset of eigenvector fields with large enough gradients. For any pair of eigenvector 
fields $X_{i}, X_{j}$, define the associated weight $\mu_{i j}$ as

$$
\mu_{i j}:=\left(f_{B_{\delta_{0} R}(z)}\left\langle X_{i}, X_{i}\right\rangle\right)^{-1 / 2}\left(f_{B_{\delta_{0} R}(z)}\left\langle X_{j}, X_{j}\right\rangle\right)^{-1 / 2} .
$$

which depends on $\delta_{0} R$. For $v \in T_{z} M$ of unit length and $c_{0}>0$, define

$$
\Lambda_{E}\left(v, z, R, \delta_{0}, c_{0}\right):=\left\{\lambda_{i}, \lambda_{j}:\left|\nabla_{v}\left\langle X_{i}, X_{j}\right\rangle(z)\right| \geq \frac{c_{0}}{R} \mu_{i j}^{-1}\right\} .
$$

Note that for a given $v$, when $c_{0}$ is chosen small enough, $\Lambda_{E}$ and $\Lambda_{L}(A) \cap \Lambda_{H}\left(A^{\prime}\right) \cap$ $\Lambda_{E}\left(v, z, R, \delta_{0}, c_{0}\right)$ are not empty. The truncated series in Lemma 11 can be slightly modified as the following so that the condition of $\Lambda_{E}\left(v, z, R, \delta_{0}, c_{0}\right)$ is included.

Lemma 12. Under Assumption 4, let $A$ and $A^{\prime}$ be chosen as in Lemma 11. For $c_{0}=$ $c_{0}\left(n, Q, \kappa,\|g\|_{C^{1, \alpha}}, \delta_{1}, \delta_{1}\right)$ small enough, there exist $c=c\left(n, Q, \kappa,\|g\|_{C^{1, \alpha}}, \delta_{0}, \delta_{1}, A, A^{\prime}, c_{0}\right)$ and $C=C\left(n, Q, \kappa,\|g\|_{C^{1, \alpha}}, \delta_{0}, \delta_{1}, A, A^{\prime}, c_{0}\right)$ so that for $v \in T_{z} M$ of unit length parallel to $\exp _{z}^{-1}(z)$, we have

$$
\left|\nabla_{v}\left\|k_{T M}(t, w, z)\right\|_{H S}^{2}\right| \sim_{c}^{C}\left|\sum_{i, j \in \Lambda} e^{-\left(\lambda_{i}+\lambda_{j}\right) t} \nabla_{v}\left\langle X_{i}(z), X_{j}(z)\right\rangle\left\langle X_{i}(w), X_{j}(w)\right\rangle\right|,
$$

where $\Lambda:=\Lambda_{L}(A) \cap \Lambda_{H}\left(A^{\prime}\right) \cap \Lambda_{E}\left(v, z, R_{z}, \delta_{0}, c_{0}\right)$ and $c, C \rightarrow 1$ when $c_{0} \rightarrow 0$.

Proof. Let $\Lambda^{c}=\Lambda_{L}(A) \cap \Lambda_{H}\left(A^{\prime}\right) \cap\left(\Lambda_{E}\left(v, z, R_{z}, \delta_{0}, c_{0}\right)\right)^{c}$. We have

$$
\begin{aligned}
& \left|\sum_{i, j \in \Lambda^{c}} e^{-\left(\lambda_{i}+\lambda_{j}\right) t} \nabla_{v}\left\langle X_{i}(z), X_{j}(z)\right\rangle\left\langle X_{i}(w), X_{j}(w)\right\rangle\right| \\
\leq & \left(\sum_{i, j \in \Lambda^{c}} e^{-\left(\lambda_{i}+\lambda_{j}\right) t}\left|\left\langle X_{i}(w), X_{j}(w)\right\rangle\right|^{2}\right)^{1 / 2}\left(\sum_{i, j \in \Lambda^{c}} e^{-\left(\lambda_{i}+\lambda_{j}\right) t}\left|\nabla_{v}\left\langle X_{i}(z), X_{j}(z)\right\rangle\right|^{2}\right)^{1 / 2} \\
\leq & k_{M}(t, w, w)^{1 / 2}\left(\sum_{i, j \in \Lambda^{c}} e^{-\left(\lambda_{i}+\lambda_{j}\right) t} \frac{c_{0}^{2}}{R^{2}} f_{B}\left\langle X_{i}, X_{i}\right\rangle f_{B}\left\langle X_{j}, X_{j}\right\rangle\right)^{1 / 2} \\
\leq & \frac{c_{0}}{R} k_{M}(t, z, z)^{1 / 2}\left(\int_{M} k_{M}(t, x, x)\right)^{1 / 2} f_{B} k_{M}\left(t, w^{\prime}, w^{\prime}\right)^{2},
\end{aligned}
$$

where the first inequality holds due to the Cauchy-Schwartz inequality, the second inequality holds by the fact that

$$
\sum_{i, j \in \Lambda^{c}} e^{-\left(\lambda_{i}+\lambda_{j}\right) t}\left|\left\langle X_{i}(w), X_{j}(w)\right\rangle\right|^{2} \leq\left\|k_{T M}(t, w, w)\right\|_{H S} \leq k_{M}(t, w, w)
$$

and

$$
\sum_{i, j \in \Lambda^{c}} e^{-\left(\lambda_{i}+\lambda_{j}\right) t}\left|\nabla_{v}\left\langle X_{i}(z), X_{j}(z)\right\rangle\right| \leq \sum_{i, j \in \Lambda^{c}} e^{-\left(\lambda_{i}+\lambda_{j}\right) t} \frac{c_{0}^{2}}{R^{2}} f_{B}\left\langle X_{i}, X_{i}\right\rangle f_{B}\left\langle X_{j}, X_{j}\right\rangle
$$

by the constraint (3.87) of $\Lambda_{E}\left(v, z, R_{z}, \delta_{0}, c_{0}\right)$, and the last inequality follows from the Kato's type inequality and similar arguments as in (3.78). This inequality proves (3.88) by reducing $c_{0}$. 
Lemma 13. Let $A, A^{\prime}$ and $c_{0}$ be chosen as in Lemma 11 and Lemma 12 under Assumption 4. For any pair $\lambda_{i}, \lambda_{j} \in \Lambda_{E}\left(v, z, R, \delta_{0}, c_{0}\right)$, there exist constants $C_{1}=C_{1}\left(c_{0}\right)$, $C_{2}=C_{2}\left(n, Q, \kappa,\|g\|_{C^{1, \alpha}}, c_{0}\right)$, and $\tau=\tau\left(n, Q, \kappa,\|g\|_{C^{1, \alpha}}, c_{0}\right)$ independent of $i, j$ so that

$$
C_{1} R^{-1} \mu_{i j}^{-1} \leq\left|\nabla_{P_{z}^{z^{\prime}} v}\left\langle X_{i}, X_{j}\right\rangle\left(z^{\prime}\right)\right| \leq C_{2} R^{-1} \mu_{i j}^{-1} .
$$

Moreover, for sufficiently large $A$ and sufficiently small $A^{\prime}$ chosen in Lemma 11, there exist $\lambda_{i}, \lambda_{j} \in \Lambda_{L}(A) \cap \Lambda_{H}\left(A^{\prime}\right) \cap \Lambda_{E}\left(v, z, R, \delta_{0}, c_{0}\right)$ so that

$$
\mu_{i j} \leq C\left(n, \kappa, D, \delta_{0}, \delta_{1}\right)
$$

Proof. The upper bound in (3.91) follows straightforward from inequalities (3.57); the lower bound in (3.91) comes directly from (3.58) and the definition of $\Lambda_{E}\left(v, z, R, \delta_{0}, c_{0}\right)$ in (3.87). Indeed, we have

$$
\begin{aligned}
\left|\nabla_{P z^{z^{\prime}} v}\left\langle X_{i}, X_{j}\right\rangle\left(z^{\prime}\right)\right| & \geq\left|\nabla_{v}\left\langle X_{i}, X_{j}\right\rangle(z)\right|-\left|\nabla_{P_{z}^{z^{\prime}} v}\left\langle X_{i}, X_{j}\right\rangle\left(z^{\prime}\right)-\nabla_{v}\left\langle X_{i}, X_{j}\right\rangle(z)\right| \\
& \geq \frac{c_{0}}{R} \mu_{i j}^{-1}-\frac{C d_{g}\left(z, z^{\prime}\right)^{\alpha}}{R^{1+\alpha}} \mu_{i j}^{-1}
\end{aligned}
$$

which leads to the lower bound if $\tau$ is chosen small enough.

Now we show (3.92). Denote $\Lambda:=\Lambda_{L}(A) \cap \Lambda_{H}\left(A^{\prime}\right) \cap \Lambda_{E}\left(v, z, R, \delta_{0}, c_{0}\right)$ and $B:=$ $B_{\delta_{0} R}(z)$. From previous Lemmas and (3.91), we obtain

$$
\begin{aligned}
& \left|\nabla_{v}\left\|k_{T M}(t, w, z)\right\|_{H S}^{2}\right| \\
\sim_{c}^{C} \mid & \sum_{\lambda_{i}, \lambda_{j} \in \Lambda} e^{-\left(\lambda_{i}+\lambda_{j}\right) t}\left\langle X_{i}(w), X_{j}(w)\right\rangle \nabla_{v}\left\langle X_{i}(z), X_{j}(z)\right\rangle \mid \\
\leq & C_{2} R^{-1} \sum_{\lambda_{i}, \lambda_{j} \in \Lambda} e^{-\left(\lambda_{i}+\lambda_{j}\right) t}\left|\left\langle X_{i}(w), X_{j}(w)\right\rangle\right| \mu_{i j}^{-1}
\end{aligned}
$$

where the approximation is by (3.68) and the inequality is by (3.91), which by the Cauchy-Schwarz inequality is bounded by

$$
C_{2} R^{-1}\left(\sum_{\lambda_{i}, \lambda_{j} \in \Lambda} e^{-2\left(\lambda_{i}+\lambda_{j}\right) t}\left\langle X_{i}(w), X_{j}(w)\right\rangle^{2}\right)^{1 / 2}\left(\sum_{\lambda_{i}, \lambda_{j} \in \Lambda} \mu_{i j}^{-2}\right)^{1 / 2}
$$

Hence,

$$
\begin{aligned}
\left|\nabla_{v}\left\|k_{T M}(t, z, w)\right\|_{H S}^{2}\right| & \leq C_{2} R^{-1}\left\|k_{T M}(2 t, w, w)\right\|_{H S}\left(\sum_{\lambda_{i}, \lambda_{j} \in \Lambda} \mu_{i j}^{-2}\right)^{1 / 2} \\
& \leq C_{2} R^{-1} k_{M}(2 t, w, w)\left(\sum_{\lambda_{i}, \lambda_{j} \in \Lambda} \mu_{i j}^{-2}\right)^{1 / 2}
\end{aligned}
$$


The bound (3.96), together with the estimate (3.66), imply that

$$
t^{-n} \frac{R}{t} \leq C R^{-1} t^{-n / 2}\left(\sum_{\lambda_{i}, \lambda_{j} \in \Lambda} \mu_{i j}^{-2}\right)^{1 / 2}
$$

and thus,

$$
t^{-n}\left(\frac{R^{2}}{t}\right)^{2} \leq C \sum_{\lambda_{i}, \lambda_{j} \in \Lambda} \mu_{i j}^{-2} .
$$

On the other hand, it is known that [44, Lemma 4.2] there exists a constant $B(n, \kappa, D)$ dependent only on $n, \kappa$ and $D$ such that for $T>0$, the following inequality holds:

$$
\#\left\{j: 0 \leq \lambda_{j} \leq T\right\} \leq e n+B(n, \kappa, D) T^{n / 2} .
$$

Here $e$ is the Euler's number.

Since $\lambda_{i}, \lambda_{j} \leq A t^{-1}$, it follows from (3.97), 3.98 that there exist some $i, j$ such that

$$
t^{-n}\left(\frac{R^{2}}{t}\right)^{2} \leq C\left(e n+B(n, \kappa, D)\left(A t^{-1}\right)^{n / 2}\right)^{2} \mu_{i j}^{-2}
$$

That is,

$$
\mu_{i j} \leq C\left(e n+B(n, \kappa, D)\left(A t^{-1}\right)^{n / 2}\right) t^{n / 2} \frac{t}{R^{2}}<C\left(n, Q, \kappa,\|g\|_{C^{1, \alpha}}, D, \delta_{0}, \delta_{1}\right)
$$

since $t \leq \delta_{1} R_{z}$. Thus, we have the uniform bound (3.92). Note that the diameter upper bound can be controlled by the volume upper bound and injectivity radius lower bound. Hence,

$$
\mu_{i j} \leq C\left(n, Q, \kappa, V, i_{0}, \delta_{1}\right)
$$

With the above Lemmas, we are now ready to prove Theorem 5 .

Theorem 5 (Parametrization via eigenvector fields for manifolds). Let $\left(M^{n}, g\right)$ be a smooth closed manifold with smooth metric $g$, Ricci curvature bound $\kappa$ and diameter upper bound D. Fix $z \in M$ and assume that Assumption 4 holds. For $R \leq R_{z}$, there exist a constant $\tau=\tau\left(n, Q, \kappa, i_{0}, \alpha, \delta_{1}\right)>1$, and $n$ pairs of indices $\left(i_{1}, j_{1}\right), \cdots,\left(i_{n}, j_{n}\right)$ so that the map

$$
\begin{aligned}
\Phi_{z}: B_{\tau^{-1} R}(z) & \rightarrow \mathbb{R}^{n} \\
x & \mapsto\left(\left\langle X_{i_{1}}, X_{j_{1}}\right\rangle(x), \cdots,\left\langle X_{i_{n}}, X_{j_{n}}\right\rangle(x)\right),
\end{aligned}
$$

is a parametrization of $B_{\tau^{-1} R}(z)$, where the associated eigenvalues satisfy

$$
\tau^{-1} R^{-2} \leq \lambda_{i_{1}}, \cdots, \lambda_{i_{n}}, \lambda_{j_{1}}, \cdots, \lambda_{j_{n}} \leq \tau R^{-2} .
$$

Furthermore, if $\Phi_{z}$ is weighted properly by

$$
\begin{aligned}
\tilde{\Phi}_{z}: B_{\tau^{-1} R}(z) & \rightarrow \mathbb{R}^{n} \\
x & \mapsto\left(\mu_{1}\left\langle X_{i_{1}}, X_{j_{1}}\right\rangle(x), \cdots, \mu_{n}\left\langle X_{i_{n}}, X_{j_{n}}\right\rangle(x)\right),
\end{aligned}
$$


where

$$
\mu_{k}:=\mu_{i_{k} j_{k}}=\left(f_{B_{\tau^{-1} R}(z)}\left\|X_{i_{k}}\right\|_{g}^{2}\right)^{-1 / 2}\left(f_{B_{\tau^{-1} R}(z)}\left\|X_{j_{k}}\right\|_{g}^{2}\right)^{-1 / 2}
$$

then for any $x, y \in B_{\tau^{-1} R}(z)$, we have

$$
\frac{1}{\tau R} d_{g}(x, y) \leq\|\tilde{\Phi}(x)-\tilde{\Phi}(y)\|_{\mathbb{R}^{n}} \leq \frac{\tau}{R} d_{g}(x, y)
$$

Here, the constants $\mu_{k}, k=1, \cdots, n$, satisfy

$$
\mu_{k} \leq C
$$

where $C$ is a constant dependent only on $n, Q, \kappa, \alpha, i_{0}$, and $V$.

Proof. Take $t, \delta_{0}$ in Lemma 11 and $c_{0}$ chosen in Lemma 12 . To simplify the notation, denote $\mu_{l}:=\mu_{i_{l}, j_{l}}$, where $l=1, \ldots, d$. Pick an arbitrary unit vector $v_{1}$. It follows from Lemma 13 that there exist $i_{1}, j_{1} \in \Lambda_{L}(A) \cap \Lambda\left(A^{\prime}\right) \cap \Lambda_{E}\left(v_{1}, z, R, \delta_{0}, c_{0}\right)$ such that $\mu_{1} \leq C\left(d, \kappa, D, \delta_{0}, \delta_{1}, A, A^{\prime}, g\right)$ and

$$
\left|\mu_{1} \nabla_{v_{1}}\left\langle X_{i_{1}}, X_{j_{1}}\right\rangle(z)\right| \geq \frac{C_{1}}{R} .
$$

Let $v_{2}$ be a unit vector orthogonal to $\nabla\left\langle X_{i_{1}}, X_{j_{1}}\right\rangle(z)$. Applying Lemma 13 again, we find another pair of indices $i_{2}, j_{2} \in \Lambda_{L}(A) \cap \Lambda_{H}\left(A^{\prime}\right) \cap \Lambda_{E}\left(v_{2}, z, R, \delta_{0}, c_{0}\right)$ so that $\mu_{2} \leq C\left(n, \kappa, D, \delta_{0}, \delta_{1}, A, A^{\prime}, g\right)$ and

$$
\left|\mu_{2} \nabla_{v_{2}}\left\langle X_{i_{2}}, X_{j_{2}}\right\rangle(z)\right| \geq \frac{C_{1}}{R}
$$

Note that by the choice of $v_{2}$, we have $\nabla_{v_{2}}\left\langle X_{i_{1}}, X_{j_{1}}\right\rangle(z)=0$, and hence $\left(i_{1}, j_{1}\right) \neq\left(i_{2}, j_{2}\right)$. We could proceed and find $v_{3}$ and so on. Suppose that we have chosen $k$ vectors $v_{1}, v_{2}, \cdots, v_{k}, k<n$, and the corresponding indices $\left(i_{1}, j_{1}\right),\left(i_{2}, j_{2}\right), \cdots,\left(i_{k}, j_{k}\right)$ such that

$$
\left|\mu_{l} \nabla_{v_{l}}\left\langle X_{i_{l}}, X_{j_{l}}\right\rangle(z)\right| \geq \frac{C_{1}}{R} \quad \text { for all } l=1, \cdots, k .
$$

Pick another unit vector $v_{k+1}$ so that $v_{k+1}$ is orthogonal to $\left\{\nabla\left\langle X_{i_{1}}, X_{j_{1}}\right\rangle(z)\right\}_{l=1}^{k}$. By Lemma 13, we have $i_{k+1}, j_{k+1} \in \Lambda_{L}(A) \cap \Lambda_{H}\left(A^{\prime}\right) \cap \Lambda_{E}\left(v_{k+1}, z, R, \delta_{0}, c_{0}\right)$ so that $\mu_{k+1} \leq$ $C\left(n, \kappa, \bar{D}, \delta_{0}, \delta_{1}, A, A^{\prime}, g\right)$ and

$$
\left|\mu_{k+1} \nabla_{v_{k+1}}\left\langle X_{i_{k+1}}, X_{j_{k+1}}\right\rangle(z)\right| \geq \frac{C_{1}}{R}
$$

We now claim $\left\{v_{1}, v_{2}, \cdots, v_{k+1}\right\}$ is linearly independent. To show this, we assume that

$$
a^{1} v_{1}+a^{2} v_{2}+\cdots+a^{k+1} v_{k+1}=0 .
$$

Consider the $(k+1) \times(k+1)$ matrix

$$
A_{k+1}:=\left(\mu_{m} \nabla_{v_{n}}\left\langle X_{i_{m}}, X_{j_{m}}\right\rangle(z)\right)_{m, n=1, \cdots, k+1} .
$$


On one hand, by 3.106$)$, we have

$$
\begin{aligned}
A_{k+1}\left(\begin{array}{c}
a^{1} \\
a^{2} \\
\vdots \\
a^{k+1}
\end{array}\right) & =a^{1}\left(\begin{array}{c}
\mu_{1} \nabla_{v_{1}}\left\langle X_{i_{1}}, X_{j_{1}}\right\rangle \\
\vdots \\
\mu_{k+1} \nabla_{v_{1}}\left\langle X_{i_{k+1}}, X_{j_{k+1}}\right\rangle
\end{array}\right)+\cdots+a^{k+1}\left(\begin{array}{c}
\mu_{1} \nabla_{v_{k+1}}\left\langle X_{i_{1}}, X_{j_{1}}\right\rangle \\
\vdots \\
\mu_{k+1} \nabla_{v_{k+1}}\left\langle X_{i_{k+1}}, X_{j_{k+1}}\right\rangle
\end{array}\right) \\
& =\left(\begin{array}{c}
\left\langle a^{1} v_{1}+\cdots+a^{k+1} v_{k+1}, \mu_{1} \nabla\left\langle X_{i_{1}}, X_{j_{1}}\right\rangle(z)\right\rangle \\
\vdots \\
\left\langle a^{1} v_{1}+\cdots+a^{k+1} v_{k+1}, \mu_{k+1} \nabla\left\langle X_{i_{k+1}}, X_{j_{k+1}}\right\rangle(z)\right\rangle
\end{array}\right)=0 .
\end{aligned}
$$

On the other hand, the matrix $A_{k+1}$ is lower-triangular since $\nabla_{v_{n}}\left\langle X_{i_{m}}, X_{j_{m}}\right\rangle=0$, for all $n>m$, which follows by the choice of the vectors $\left\{v_{l}\right\}_{l=1}^{k+1}$. Therefore $a^{l}=0$ for all $l=1, \cdots, k+1$ and hence $\left\{v_{1}, v_{2}, \cdots, v_{k+1}\right\}$ is linearly independent.

With the above chosen eigenvector fields, we now show the embedding propoerty. For any $x_{1}, x_{2} \in B_{\delta_{0} R}(z)$, let $\gamma(t), 0 \leq t \leq 1$ be the geodesic curve joining $x_{1}=\gamma(0)$ and $x_{2}=\gamma(1)$. Under the harmonic coordinate chart $(\phi, U), \phi: U \subset \mathbb{R}^{n} \rightarrow M$ so that $\phi(0)=z$ and $g_{a b}(0)=\delta_{a b}$, we have $\gamma(t)=\phi(\tilde{\gamma}(t))$ for some curve $\tilde{\gamma}(t) \subset U$. We also assume that $\left\|\tilde{\gamma}^{\prime}(t)\right\|_{g}=d_{g}\left(x_{1}, x_{2}\right)$. Using the basis $\left\{v_{l}\right\}_{l=1}^{n} \subset T_{z} M \cong \mathbb{R}^{n}$, we have

$$
\tilde{\gamma}^{\prime}(t)=\sum_{l=1}^{n} a^{l}(t) v_{l} \quad \text { and } \quad \gamma^{\prime}(t)=\left.\sum_{l=1}^{n} a^{l}(t) d \phi\right|_{\tilde{\gamma}(t)}\left(v_{l}\right)
$$

We claim that there exists $c>0$ so that

$$
\left\|\left.\nabla \tilde{\Phi} \circ \phi\right|_{0} \tilde{\gamma}^{\prime}(t)\right\|_{\mathbb{R}^{d}} \geq \frac{c}{R}\left\|\tilde{\gamma}^{\prime}(t)\right\|_{g}=\frac{c}{R}
$$

that is, the geodesic deformation has a lower bound. We prove this statement by contradiction. Denote $\tilde{\Phi}_{k}:=\left(\mu_{1}\left\langle X_{i_{1}}, X_{j_{1}}\right\rangle, \cdots, \mu_{k}\left\langle X_{i_{k}}, X_{j_{k}}\right\rangle\right)$ so that $\tilde{\Phi}=\tilde{\Phi}_{d}$. Suppose that for all $k=1, \cdots, n$,

$$
\left\|\left.\nabla \tilde{\Phi}_{k} \circ \phi\right|_{0} \frac{d}{d t} \tilde{\gamma}(t)\right\|_{\mathbb{R}^{k}} \leq \frac{c_{m}}{R}
$$

for any small constant $c_{m}$. We have, by the local parametrization 3.109,

$$
\left\|\left.\nabla \tilde{\Phi}_{k} \circ \phi\right|_{0} \tilde{\gamma}^{\prime}(t)\right\|_{\mathbb{R}^{k}}=\left\|\left.\sum_{l=1}^{n} a^{l}(t) \nabla \tilde{\Phi}_{k} \circ \phi\right|_{0} v_{l}\right\|_{\mathbb{R}^{k}}=\left\|\sum_{l=1}^{n} a^{l} \nabla_{v_{l}} \tilde{\Phi}_{k}(z)\right\|_{\mathbb{R}^{k}} .
$$

Since $\nabla_{v_{l}}\left\langle X_{i_{m}}, X_{j_{m}}\right\rangle=0$ for all $m<l$, the first $k$ terms are the only non-zero terms, that is,

$$
\left\|\left.\nabla \tilde{\Phi}_{k} \circ \phi\right|_{0} \tilde{\gamma}^{\prime}(t)\right\|_{\mathbb{R}^{k}}=\left\|\sum_{l=1}^{k} a^{l} \nabla_{v_{l}} \tilde{\Phi}_{k}(z)\right\|_{\mathbb{R}^{k}}
$$


We further note that

$$
\begin{aligned}
\left\|\sum_{l=1}^{k} a^{l} \nabla_{v_{l}} \tilde{\Phi}_{k}(z)\right\|_{\mathbb{R}^{k}} & \geq\left|a^{k} \mu_{k} \nabla_{v_{k}}\left\langle X_{i_{k}}, X_{j_{k}}\right\rangle(z)\right|-\sum_{l=1}^{k-1}\left\|a^{l} \nabla_{v_{l}} \tilde{\Phi}_{k}(z)\right\|_{\mathbb{R}^{k}} \\
& \geq \frac{c_{0}}{R}\left|a^{k}\right|-\frac{c}{R} \sum_{l=1}^{k-1}\left|a^{l}\right|
\end{aligned}
$$

since, by the choice of eigenvectors fields, $\mu_{k} \nabla_{v_{k}}\left\langle X_{i_{k}}, X_{j_{k}}\right\rangle(z) \geq \frac{c_{0}}{R}$ and, by (3.57) in Corollary 11, $\mu_{i j}\left|\nabla_{v_{l}}\left\langle X_{i}, X_{j}\right\rangle(z)\right| \leq \frac{c}{R}$. By the assumption (3.111), we have

$$
\frac{c_{0}}{R}\left|a^{k}\right|-\frac{c}{R} \sum_{l=1}^{k-1}\left|a^{l}\right| \leq\left\|\left.\nabla \tilde{\Phi}_{k} \circ \phi\right|_{0} \frac{d}{d t} \tilde{\gamma}(t)\right\|_{\mathbb{R}^{k}} \leq \frac{c_{m}}{R}
$$

By induction, we now show that

$$
\left|a^{k}\right| \leq \frac{c_{m}}{c_{0}}\left(\frac{c}{c_{0}}+1\right)^{k-1}
$$

For $k=1,(3.116)$ follows from $(3.115)$. Suppose that 3.116$)$ is true for $k=l$. Then

$$
\begin{aligned}
\left|a^{l+1}\right| & \leq \frac{c_{m}}{c_{0}}+\frac{c}{c_{0}} \sum_{i=1}^{l}\left|a^{i}\right| \\
& \leq \frac{c_{m}}{c_{0}}+\frac{c}{c_{0}} \sum_{i=1}^{l} \frac{c_{m}}{c_{0}}\left(\frac{c}{c_{0}}+1\right)^{i-1} \\
& =\frac{c_{m}}{c_{0}}\left(1+\frac{c}{c_{0}} \frac{\left(\frac{c}{c_{0}}+1\right)^{l}-1}{\frac{c}{c_{0}}+1-1}\right)=\frac{c_{m}}{c_{0}}\left(\frac{c}{c_{0}}+1\right)^{l}
\end{aligned}
$$

and we have (3.116). The inequality $(3.116)$ implies $\left|a^{l}\right|$ is arbitrarily small for all $l=1, \cdots, n$ for $c_{m}$ sufficiently small, which leads to a contradiction since $\left\|\tilde{\gamma}^{\prime}(t)\right\|_{g}=$ $d_{g}\left(x_{1}, x_{2}\right)$ is constant.

By Corollary 9, we also have

$$
\left\|\left.\nabla \tilde{\Phi}\right|_{x}-\left.\nabla \tilde{\Phi}\right|_{z}\right\|_{o p} \leq C\left(\frac{d_{g}(x, z)}{R}\right)^{\alpha} \frac{1}{R} \leq C \delta_{0}^{\alpha} \frac{1}{R}
$$

which is small when $\delta_{0}$ is small enough. 
With the above preparation, the lower bound in 3.5 follows by the bounds 3.110 and (3.118). Indeed, we have

$$
\begin{aligned}
& \left|\tilde{\Phi}\left(x_{2}\right)-\tilde{\Phi}\left(x_{1}\right)\right| \\
= & \left|\int_{0}^{1} \nabla(\tilde{\Phi} \circ \phi)_{\tilde{\gamma}(t)} \frac{d}{d t} \tilde{\gamma}(t) d t\right| \\
= & \left|\int_{0}^{1} \nabla \tilde{\Phi} \circ \phi\right|_{0} \frac{d}{d t} \tilde{\gamma}(t)+\left(\left.\nabla \tilde{\Phi} \circ \phi\right|_{\tilde{\gamma}(t)}-\left.\nabla \tilde{\Phi} \circ \phi\right|_{0}\right) \frac{d}{d t} \tilde{\gamma}(t) d t \mid \\
\geq & \int_{0}^{1} \frac{c}{R}\left\|\tilde{\gamma}^{\prime}(t)\right\|_{g} d t=\frac{c}{R} d_{M}\left(x_{1}, x_{2}\right),
\end{aligned}
$$

where the last inequality holds due to 3.110 and $(3.118)$ when $\delta_{0}$ is small enough.

To finish the proof of the low distortion property $(3.5)$ of the mapping $\tilde{\Phi}_{z}$, we should the upper bound in (3.5). Note that b Corollary 9 , for all $k, l=1, \cdots, n$,

$$
\left|\mu_{k} \nabla_{v_{l}}\left\langle X_{i_{k}}, X_{j_{k}}\right\rangle\right|_{\gamma(t)} \mid \leq \frac{C_{2}}{R}
$$

which leads to the upper bound in 3.5 ,

$$
\left\|\tilde{\Phi}\left(x_{2}\right)-\tilde{\Phi}\left(x_{1}\right)\right\|_{\mathbb{R}^{n}}=\left\|\left.\int_{0}^{1} \nabla \tilde{\Phi}\right|_{\gamma(t)} \gamma^{\prime}(t) d t\right\|_{\mathbb{R}^{n}} \leq \frac{C_{2}}{R} d_{g}\left(x_{1}, x_{2}\right) .
$$

\section{Manifold Embedding by Truncated Vector Diffusion Map}

The goal in this section is to prove Theorem 1 under the assumption of the smooth metric; that is, we could embed manifolds in $\mathcal{M}_{n, \kappa, i_{0}, V}$ into $\mathbb{R}^{N^{2}}$ for some finite $N$, where $N$ depends only on $n, \kappa, i_{0}$ and $V$. To find $n$ and the embedding for $\mathcal{M}_{n, \kappa, i_{0}, V}$, we need two lemmas. First, in Lemma 14, we show that based on Theorem 1, for any $M \in \mathcal{M}_{n, \kappa, i_{0}, V}, M$ can be immersed into an Euclidean space. Note that we have to link the conditions of $\mathcal{M}_{n, \kappa, i_{0}, V}$ back to the assumptions for Theorem 1. Second, in Lemma 15 we control the remainder term of the series expansion of $\left\|k_{T M}(t, w, z)\right\|_{H S}^{2}$; together with the upper bound of $\left\|k_{T M}(t, w, z)\right\|_{H S}^{2}$, the immersion is improved to the embedding and we finish the proof of Theorem 1 .

Lemma 14. For any $M \in \mathcal{M}_{n, \kappa, i_{0}, V}$ and any $z \in M$, there exist a positive integer $m$ depending only on $n, \kappa, i_{0}, V$ and $\tau$ and a constant $\epsilon>0$ so that

$$
\begin{aligned}
\Phi_{z}^{m^{2}}: B_{\epsilon}(z) & \rightarrow \mathbb{R}^{m^{2}} \\
x & \mapsto\left(\left\langle X_{i}(x), X_{j}(x)\right\rangle\right)_{i, j=1}^{m}
\end{aligned}
$$

is a smooth embedding.

Proof. We control the eigenvalues by applying the part (a) of Lemma 4.2 in [44]. To do so, note that for each $M \in \mathcal{M}_{n, \kappa, i_{0}, V}$, since $\operatorname{Vol}(M)$ is bounded by $V$ from above and the injectivity is bounded by $i_{0}$ from below, by the packing argument, the diameter of 
$M$ is bounded from above [3, p. 267]. Thus, by the part (a) of Lemma 4.2 in [44], there exists a positive constant $A\left(n, \kappa, i_{0}, V\right)$ so that

$$
\lambda_{j} \geq A\left(n, \kappa, i_{0}, V\right) j^{2 / n} .
$$

Choose $m$ be the smallest integer so that

$$
A\left(n, \kappa, i_{0}, V\right)(m+1)^{2 / n}>\tau r_{h}^{-2},
$$

where $\tau=\tau(n, Q)$ is the constant chosen in Theorem 5. Therefore, following (4.2),

$$
\lambda_{m+1} \geq A\left(n, \kappa, i_{0}, V\right)(m+1)^{2 / n}>\tau r_{h}^{-2},
$$

for any $M \in \mathcal{M}_{n, \kappa, i_{0}, V}$. On the other hand, by Theorem 5 , for a given $M \in \mathcal{M}_{n, \kappa, i_{0}, V}$, there are $n$ pairs of indices $\left(i_{1}, j_{1}\right), \cdots,\left(i_{n}, j_{n}\right)$ so that the map

$$
\begin{aligned}
\Phi_{z}: B_{\tau^{-1} r_{h}}(z) & \rightarrow \mathbb{R}^{n} \\
x & \mapsto\left(\left\langle X_{i_{1}}, X_{j_{1}}\right\rangle(x), \cdots,\left\langle X_{i_{n}}, X_{j_{n}}\right\rangle(x)\right),
\end{aligned}
$$

is an embedding, where $x \in B_{\tau^{-1} r_{h}}(z)$ and

$$
\tau^{-1} r_{h}^{-2} \leq \lambda_{i_{1}}, \cdots, \lambda_{i_{n}}, \lambda_{j_{1}}, \cdots, \lambda_{j_{n}} \leq \tau r_{h}^{-2}
$$

Since $m$ is a universal natural number chosen to satisfy $\lambda_{m+1}>\tau r_{h}^{-2}$, by (4.6), we conclude that the mapping $\Phi_{z}^{m^{2}}: B_{\epsilon}(z) \rightarrow \mathbb{R}^{m^{2}}$ is an embedding with $\epsilon=\tau^{-1} r_{h}$.

In the next Lemma, we would control the difference between the diagonal terms of the heat kernel and its truncation.

Lemma 15. Take $M \in \mathcal{M}_{n, \kappa, i_{0}, V}$. Denote

$$
R_{k}(t):=\sup _{x \in M} \sum_{i \text { or } j \geq k} e^{-\left(\lambda_{i}+\lambda_{j}\right) t}\left\langle X_{i}(x), X_{j}(x)\right\rangle^{2} .
$$

For all $k \in \mathbb{N}$, there is a function $E_{k}(t): \mathbb{R}^{+} \rightarrow \mathbb{R}^{+}$such that for all $M \in \mathcal{M}_{n, \kappa, i_{0}, V}$,

$$
R_{k}(t) \leq E_{k}(t) t^{-(3 n / 2+1)}
$$

and $\lim _{k \rightarrow \infty} E_{k}(t)=0$ for a fixed $t>0$.

Proof. It suffices to prove that

$$
\sum_{i \geq k, j} e^{-\left(\lambda_{i}+\lambda_{j}\right) t}\left\langle X_{i}(x), X_{j}(x)\right\rangle^{2} \leq E_{k}(t) t^{-n} .
$$

Consider the positive measure

$$
d \mu_{x}(\lambda, \nu)=\sum_{i \geq k, j}\left\langle X_{i}(x), X_{j}(x)\right\rangle^{2} \delta_{\lambda_{i}} \times \delta_{\lambda_{j}}
$$


where $\delta_{\lambda_{i}}$ is the Dirac measure at $\lambda_{i} \in \mathbb{R}$. By a direct calculation, we have

$$
\begin{aligned}
e^{-(\lambda+\nu) t} \sum_{i \geq k, j}\left\langle X_{i}(x), X_{j}(x)\right\rangle^{2} & =\int_{\mathbb{R}} \int_{\mathbb{R}} e^{-(\lambda+\nu) t} d \mu_{x}(\lambda, \nu) \\
& =\int_{\mathbb{R}} \int_{\mathbb{R}}\left(\partial_{\lambda} \partial_{\nu} e^{-(\lambda+\nu) t}\right) \sum_{\lambda_{k} \leq \lambda_{i} \leq \lambda, \lambda_{j} \leq \nu}\left\langle X_{i}(x), X_{j}(x)\right\rangle^{2} d \lambda d \nu,
\end{aligned}
$$

where the last equality follows by the definition of the derivative in the sense of distribution. Hence,

$$
\begin{aligned}
e^{-(\lambda+\nu) t} \sum_{i \geq k, j}\left\langle X_{i}(x), X_{j}(x)\right\rangle^{2} & =\int_{0}^{\infty} \int_{\lambda_{k}}^{\infty} t^{2} e^{-(\lambda+\nu) t} \sum_{\lambda_{k} \leq \lambda_{i} \leq \lambda, \lambda_{j} \leq \nu}\left\langle X_{i}(x), X_{j}(x)\right\rangle^{2} d \lambda d \nu \\
& =\int_{0}^{\infty} \int_{t \lambda_{k}}^{\infty} e^{-\left(\lambda^{\prime}+\nu^{\prime}\right)} \sum_{\substack{\lambda_{k} \leq \lambda_{i} \leq \frac{\lambda^{\prime}}{t}, \lambda_{j} \leq \nu^{\prime} \\
t}}\left\langle X_{i}(x), X_{j}(x)\right\rangle^{2} d \lambda^{\prime} d \nu^{\prime},
\end{aligned}
$$

where $\lambda^{\prime}=\lambda t$ and $\nu^{\prime}=\nu t$. Since the lower bound of injectivity and the upper bound of volume imply the upper bound of the diameter, by the inequality (32) in [44], we have

$$
\sum_{\lambda_{i} \leq \frac{\lambda^{\prime}}{t}, \lambda_{j} \leq \frac{\nu^{\prime}}{t}}\left\langle X_{i}(x), X_{j}(x)\right\rangle^{2} \leq \frac{C\left(n, \kappa, i_{0}, V\right)}{\operatorname{Vol}(M)^{2}}\left(\frac{\lambda^{\prime}}{t}\right)^{n / 2}\left(\frac{\nu^{\prime}}{t}\right)^{n / 2},
$$

where $C\left(n, \kappa, i_{0}, V\right)$ is a universal constant depending only on $n, \kappa, i_{0}$ and $V$.

Since $\int_{0}^{\infty} \nu^{\prime n / 2} e^{-\nu^{\prime} t} d \nu^{\prime}=t^{-n / 2-1} \int_{0}^{\infty} x^{n / 2} e^{-x} d x=\Gamma(n / 2+1) t^{-\left(\frac{n}{2}+1\right)}$, where $\Gamma$ is the Gamma function, and $t A\left(n, \kappa, i_{0}, V\right) k^{2 / n} \leq t \lambda_{k}$ by 4.2$)$, we have

$$
\begin{aligned}
e^{-(\lambda+\nu) t} \sum_{i \geq k, j}\left\langle X_{i}(x), X_{j}(x)\right\rangle^{2} & \leq C t^{-(3 n / 2+1)} \int_{t \lambda_{k}}^{\infty} \lambda^{\prime n} e^{-\lambda^{\prime} t} d \lambda^{\prime} \\
& \leq C t^{-(3 n / 2+1)} \int_{t A\left(d, \kappa, i_{0}, V\right) k^{2 / d}}^{\infty} \lambda^{\prime n} e^{-\lambda^{\prime} t} d \lambda^{\prime} .
\end{aligned}
$$

Define

$$
E_{k}(t):=C \int_{t A\left(d, \kappa, i_{0}, V\right) k^{2 / n}}^{\infty} \lambda^{\prime n} e^{-\lambda^{\prime} t} d \lambda .
$$

Hence $R_{k}(t) \leq E_{k}(t) t^{-(3 n / 2+1)}$ and it is clear that $\lim _{k \rightarrow \infty} E_{k}(t)=0$ for a fixed $t>0$.

With the above Lemmas, we are ready to prove Theorem 1 .

Theorem 1 (Embeddings of Riemannian Manifolds with Finite Eigenvector Fields of Connection Laplacian). For a smooth closed manifold $(M, g)$ with smooth metric and for any $t>0$, there is a positive integer $N_{0}$ so that the $t V D M V_{t}^{N^{2}}$ is a smooth embedding for all $N \geq N_{0}$.

Proof. By Lemma 14, there exists a integer $m$ so that $\Phi_{z}^{m^{2}}$ is a smooth embedding for all $z \in M$ and all $M \in \mathcal{M}_{n, \kappa, i_{0}, V}$. Thus, if we choose $\tilde{m} \geq m$, we know that $\Phi^{\tilde{m}^{2}}$ is an immersion. It suffices to show that for $x, y \in M$ so that $d_{g}(x, y)>\epsilon, \Phi^{\tilde{m}^{2}}(x) \neq \Phi^{\tilde{m}^{2}}(y)$. 
By the asymptotic expansion of the heat kernel (see [33, p. 1094] and [8, p. 87]), when $t \ll 1$,

$$
\left\|k_{T M}(t, x, y)\right\|_{H S}^{2}=(4 \pi t)^{-n} e^{-\frac{\|v\|_{g}^{2}}{2 t}}\left(1-\frac{\operatorname{Ric}(v, v)}{6}+O\left(\|v\|_{g}^{3}\right)\right)(n+O(t)),
$$

where $v \in T_{x} M$ so that $y=\exp _{x}(v)$. Therefore, for all $M \in \mathcal{M}_{n, \kappa, i_{0}, V}$ and a fixed constant $\epsilon>0$, there exists a universal constant $C_{U, \epsilon}$ so that for any $x, y \in M$ such that $d_{g}(x, y) \geq \epsilon$,

$$
\left\|k_{T M}(t, x, y)\right\|_{H S}^{2} \leq \frac{C_{U, \epsilon}}{t^{n}} \exp \left(-\frac{\epsilon^{2}}{2 t}\right) .
$$

Let $c_{1}(t)=\inf _{x \in M}\left\|k_{T M}(t, x, x)\right\|_{H S}^{2}$. Clearly, $c_{1}(t)=(4 \pi t)^{-n}(n+O(t))>0$.

Let $\epsilon$ and $m$ be chosen as in Lemma 14. Define

$$
G(t):=c_{1}(t)-\sup _{d_{g}(x, y) \geq \epsilon}\left\|k_{T M}(t, x, y)\right\|_{H S}^{2} .
$$

Since $\sup _{d_{g}(x, y) \geq \epsilon}\left\|k_{T M}(t, x, y)\right\|_{H S}^{2} \leq t^{-n} \exp \left(-\frac{\epsilon^{2}}{2 t}\right) \rightarrow 0$ as $t \rightarrow 0^{+}, G(t) \rightarrow c_{1}(t)$ as $t \rightarrow 0^{+}$. By the definition of $c_{1}(t)$ and Lemma 10 , for $t \in\left(0, \frac{i_{0}}{2}\right]$,

$$
\begin{aligned}
G(t) & \leq \inf _{d_{g}(x, y) \geq \epsilon}\left\|k_{T M}(t, x, x)\right\|_{H S}^{2}-\sup _{d_{g}(x, y) \geq \epsilon}\left\|k_{T M}(t, x, y)\right\|_{H S}^{2} \\
& \leq \inf _{d_{g}(x, y) \geq \epsilon}\left(\left\|k_{T M}(t, x, x)\right\|_{H S}^{2}-\left\|k_{T M}(t, x, y)\right\|_{H S}^{2}\right) .
\end{aligned}
$$

Choose $t_{0} \in\left(0, \frac{i_{0}}{2}\right]$ so that $G\left(t_{0}\right) \geq \frac{4}{5} c_{1}\left(t_{0}\right)$. Then choose $\tilde{m} \geq m$ big enough so that $R_{\tilde{m}+1}\left(t_{0}\right) \leq \frac{1}{5} c_{1}\left(t_{0}\right)$, where $E_{\tilde{m}+1}$ is defined in 4.8). Denote the truncated heat kernel by

$$
k_{T M}^{(\tilde{m})}(t, x, y):=\sum_{i=1}^{\tilde{m}} e^{-\lambda_{i} t} X_{i}(x) \otimes X_{i}(y)
$$

so that

$$
\begin{aligned}
\left\|k_{T M}^{(\tilde{m})}(t, x, y)\right\|_{H S}^{2} & =\sum_{i, j \leq \tilde{m}} e^{-\left(\lambda_{i}+\lambda_{j}\right) t}\left\langle X_{i}(x), X_{j}(x)\right\rangle\left\langle X_{i}(y), X_{j}(y)\right\rangle \\
& =\left\langle V_{t}^{\tilde{m}^{2}}(x), V_{t}^{\tilde{m}^{2}}(y)\right\rangle .
\end{aligned}
$$

Note that

$$
\begin{aligned}
& \sup _{x, y \in M}\left(\left\|k_{T M}\left(t_{0}, x, y\right)\right\|_{H S}^{2}-\left\|k_{T M}^{(\tilde{m})}\left(t_{0}, x, y\right)\right\|_{H S}^{2}\right) \\
& =\sup _{x, y \in M_{i}} \sum_{\text {or }} e_{j \geq \tilde{m}+1} e^{-\left(\lambda_{i}+\lambda_{j}\right) t_{0}}\left\langle X_{i}, X_{j}\right\rangle(x)\left\langle X_{i}, X_{j}\right\rangle(y) \\
& \leq R_{\tilde{m}+1}\left(t_{0}\right) \leq \frac{1}{5} c_{1}\left(t_{0}\right),
\end{aligned}
$$


where the inequalities hold by the Cauchy-Schwartz inequality, (4.8) and the chosen $\tilde{m}$. With the above, we have

$$
\begin{aligned}
\frac{4}{5} c_{1}\left(t_{0}\right) & \leq G\left(t_{0}\right) \\
& \leq\left\|k_{T M}\left(t_{0}, x, x\right)\right\|_{H S}^{2}-\left\|k_{T M}\left(t_{0}, x, y\right)\right\|_{H S}^{2} \\
& \leq\left\|k_{T M}^{(\tilde{m})}\left(t_{0}, x, x\right)\right\|_{H S}^{2}-\left\|k_{T M}^{(\tilde{m})}\left(t_{0}, x, y\right)\right\|_{H S}^{2}+\frac{2}{5} c_{1}\left(t_{0}\right),
\end{aligned}
$$

where the second inequality comes from (4.19) and the last inequality holds due to 4.23. Therefore $\left\|k_{T M}^{(\tilde{m})}\left(t_{0}, x, x\right)\right\|_{H S}^{2} \neq\left\|k_{T M}^{(\tilde{m})}\left(t_{0}, x, y\right)\right\|_{H S}^{2}$, which indicates

$$
V_{t_{0}}^{\tilde{m}^{2}}(x) \neq V_{t_{0}}^{\tilde{m}^{2}}(y) \text {. }
$$

and that tVDM is an embedding. Since tVDM $V_{t}^{\tilde{m}^{2}}$ with any time $t>0$ and $V_{t_{0}}^{\tilde{m}^{2}}$ differ only by constant scalings, they are diffeomorphic. We thus conclude the proof of the Theorem.

\section{Universal Local Parametrization and Embedding for Rough MANIFOLDS}

In this section, we finish the proof of Theorem 1 when the metric is of lower regularity $g \in c^{2, \alpha}$. The key step is to approximate $g$ by smooth metrics $g_{\epsilon}$. The parametrization for $(M, g)$ can be approximated by the parametrizations for smooth manifolds $\left(M, g_{\epsilon}\right)$ defined as in Theorem 5 and hence we have Theorem 1 for $g \in c^{2, \alpha}$. The approximation relies on the $C^{1}$ bound of the eigenvector field perturbation under the $c^{2, \alpha}$ metric perturbation, which is stated in Theorem 16 below. While Theorem 16 is of independent interest, we include it here to complete the argument. The same proof could be applied to study the $C^{1}$ bound of the eigenfunction perturbation under the metric perturbation with a suitable regularity, which generalizes Theorem 21 in [7].

Suppose that metrics $g$ and $h$ on $M$ satisfy

$$
(1-\epsilon)\|g\|_{c^{2, \alpha}}<\|h\|_{c^{2, \alpha}}<(1+\epsilon)\|g\|_{c^{2, \alpha}} .
$$

By the assumption and the min-max principle, and hence the continuity of eigenvalues, there exists $\epsilon^{\prime}=O(\epsilon)$ such that for any $X \in C^{\infty}(T M)$,

$$
\begin{gathered}
\left(1-\epsilon^{\prime}\right)\|X\|_{L^{2}(g)}^{2} \leq\|X\|_{L^{2}(h)}^{2} \leq\left(1+\epsilon^{\prime}\right)\|X\|_{L^{2}(g)}^{2} \\
\left(1-\epsilon^{\prime}\right)\left\|\nabla_{g} X\right\|_{L^{2}(g)}^{2} \leq\left\|\nabla_{h} X\right\|_{L^{2}(h)}^{2} \leq\left(1+\epsilon^{\prime}\right)\left\|\nabla_{g} X\right\|_{L^{2}(g)}^{2} \\
\left(1-\epsilon^{\prime}\right) \lambda_{j}(g) \leq \lambda_{j}(h) \leq\left(1+\epsilon^{\prime}\right) \lambda_{j}(g) \text { for any } j \geq 0
\end{gathered}
$$

and By the same argument for the continuity of eigenvalues, we have that for any $X \in C^{\infty}(T M)$,

$$
\left(1-\epsilon^{\prime}\right)\left\|\nabla_{g}^{2} X\right\|_{L^{2}(g)}^{2} \leq\left\|\nabla_{h}^{2} X\right\|_{L^{2}(h)}^{2} \leq\left(1+\epsilon^{\prime}\right)\left\|\nabla_{g}^{2} X\right\|_{L^{2}(g)}^{2} .
$$


Theorem 16. Let $h$ be a metric on $M$ of dimension $n$ such that $(1-\epsilon)\|g\|_{c^{2, \alpha}}<$ $\|h\|_{c^{2, \alpha}}<(1+\epsilon)\|g\|_{c^{2, \alpha}}$. Suppose for both $(M, g)$ and $(M, h)$, the infectivity radii are bounded from below by $i_{0}>0$, the volumes are bounded above by $V>0$, and the Ricci curvatures satisfy $\mid$ Ric $\mid \leq \kappa$. Let $k_{0} \in \mathbb{N}$ be given. Then there exist constants $\eta_{g, i}(\epsilon), 1 \leq i \leq k_{0}$, which go to zero with $\epsilon$, such that for any orthonormal basis $\left\{Y_{i}\right\}$ of eigenvector fields of $\nabla_{h}^{2}$, one can associate an orthonormal basis $\left\{X_{i}\right\}$ of eigenvector fields of $\nabla_{g}^{2}$ satisfying

$$
\left\|X_{i}-Y_{i}\right\|_{L^{\infty}(B, g)} \leq \eta_{g, i, K}(\epsilon)
$$

and

$$
\left\|\nabla_{g} X_{i}-\nabla_{g} Y_{i}\right\|_{L^{\infty}(B, g)} \leq \eta_{g, i, K}(\epsilon)
$$

for all $i<k_{0}$, where $B:=B_{R}(z)$.

The associated basis $X_{i}, 1 \leq i \leq J$, are chosen by the Gram-Schmidt process. Lemma 17 and Proposition 18 control the norms of $\left\|X_{i}-Y_{i}\right\|_{H^{1}(g)}$ and $\left\|\nabla_{g}^{2} X_{i}-\nabla_{g}^{2} Y_{i}\right\|_{L^{2}(g)}$. To obtain the sup-norm bound (5.6) and (5.7), we need to control $\left\|X_{i}\right\|_{L^{\infty}(B, g)},\left\|Y_{i}\right\|_{L^{\infty}(B, h)}$, $\left\|\nabla_{g} X_{i}\right\|_{L^{\infty}(B, g)},\left\|\nabla_{g} Y_{i}\right\|_{L^{\infty}(B, h)},\left\|\nabla_{g}^{2} X_{i}\right\|_{L^{\infty}(B, g)}$, and $\left\|\nabla_{g}^{2} Y_{i}\right\|_{L^{\infty}(B, h)}$ on local balls which can be proved by Proposition 8 using Lemma 7, 6.

Let $\Lambda_{1}<\Lambda_{2}<\cdots<\Lambda_{k}<\cdots$ be the distinct eigenvalues of $\nabla_{g}^{2}$. Let $E_{k}$ and $m_{k}$ be the corresponding eigenspaces and multiplicities, and $N_{k}=m_{1}+m_{2}+\cdots+m_{k}$. By the min-max principle, the eigenvalues is continuously perturbed with respect to the deviation (5.1). Therefore, for a fixed $k_{0}$, we can find $\epsilon_{0}>0$ so that for all $\epsilon<\epsilon_{0}$ and $h$ satisfies (5.1), the first $N_{k_{0}}$ eigenvalues of $\nabla_{h}^{2}$, where we count the multiplicity, are all less than $\Lambda_{k_{0}+1}$ and contained in pairwise disjoint intervals $I_{k}$, for $1 \leq k \leq k_{0}$. Let $F_{k}, 1 \leq k \leq k_{0}$, be the sum of eigenspaces of $\nabla_{h}^{2}$ corresponding to the eigenvalues $\lambda_{j}(h)$ contained in the interval $I_{k}$ about $\Lambda_{k}$. Let $\pi_{k}$ denote the orthogonal projection in $L^{2}(g)$ onto the eigenspace $E_{k}$.

Lemma 17. Fix $k_{0}$. For $k \leq k_{0}$, there exist functions $\alpha_{k}(\epsilon), \beta_{k}(\epsilon)$, and $\gamma_{k}(\epsilon)$ which go to zero with $\epsilon$ such that

(a) $\left\|\left(\pi_{k}-I d\right) Y\right\|_{H^{1}(g)} \leq \alpha_{k}(\epsilon)\|Y\|_{L^{2}(h)} \quad$ for any $Y \in F_{k}$,

(b) For any $X \in \oplus_{i=1}^{k} E_{i}$ and for any $Y$ that is $L^{2}(h)$-orthogonal to $\oplus_{i=1}^{k} F_{i}$,

$$
\left|\langle X, Y\rangle_{g}\right| \leq \beta_{k}(\epsilon)\|X\|_{L^{2}(g)}\|Y\|_{L^{2}(h)} .
$$

(c) $\left\|\nabla_{g}^{2}\left(\pi_{k}-I d\right) Y\right\|_{L^{2}(g)} \leq \gamma_{k}(\epsilon)\|Y\|_{L^{2}(h)} \quad$ for any $Y \in F_{k}$.

Proof. The proof of assertions (a) and (b) follow straightforwardly from the proof of Lemma 20 in [7].

We prove (c) by induction. Start with $k=1$. Take $Y \in F_{1}$ with $\|Y\|_{L^{2}(h)}^{2}=1$. Write $Y=X+X^{\prime \prime}$ with $X=\pi_{1}(Y)$. Then $\nabla_{g}^{2} X$ and $\nabla_{g}^{2} X^{\prime \prime}$ are orthogonal with respect to $L^{2}(g)$ since $\nabla_{g}^{2} X \in E_{1}$ and $\nabla_{g}^{2} X^{\prime \prime} \in E_{1}^{\perp}$. By (5.5), it follows that

$$
\left(1-\epsilon^{\prime}\right)\left(\left\|\nabla_{g}^{2} X\right\|_{L^{2}(g)}+\left\|\nabla_{g}^{2} X^{\prime \prime}\right\|_{L^{2}(g)}\right) \leq\left\|\nabla_{h}^{2} Y\right\|_{L^{2}(h)}=\lambda_{1}(h) \leq \Lambda_{1}\left(1+\epsilon^{\prime}\right) .
$$

By (a), we know that $\left|\|X\|_{g}-\|Y\|_{g}\right| \leq \alpha_{1}(\epsilon)$ and $\|Y\|_{g} \leq\left(1+2 \epsilon^{\prime}\right)\|Y\|_{h}=1+2 \epsilon^{\prime}$, which leads to the fact that $\|X\|_{g}=1+O(\epsilon)$. The bounds (5.9) together with the fact 
$\left\|\nabla_{g}^{2} X\right\|_{L^{2}(g)}=\Lambda_{1}\|X\|_{L^{2}(g)}=\Lambda_{1}(1+O(\epsilon))$, when $\epsilon$ is small enough, we have

$$
\left\|\nabla_{g}^{2} X^{\prime \prime}\right\|_{L^{2}(g)}=O(\epsilon)
$$

Assume that (c) is true for $F_{1}, F_{2}, \cdots, F_{k-1}$. Define $E_{k}^{\prime}=\oplus_{i \leq k-1} E_{i}$ and $E_{k}^{\prime \prime}=\oplus_{i \geq k+1} E_{i}$. Take any $Y \in F_{k}$ such that $\|Y\|_{L^{2}(h)}=1$. Write $Y=X^{\prime}+\bar{X}+X^{\prime \prime}$ with respect to the decomposition $E_{k}^{\prime} \oplus E_{k} \oplus E_{k}^{\prime \prime}$ of $L^{2}(g)$. Note that $\nabla_{g}^{2} X^{\prime}, \nabla_{g}^{2} X$ and $\nabla_{g}^{2} X^{\prime \prime}$ are orthogonal in $L^{2}(g)$, so we have

$$
\left(1-\epsilon^{\prime}\right)\left(\Lambda_{k}\|X\|_{L^{2}(g)}+\left\|\nabla_{g}^{2} X^{\prime}\right\|_{L^{2}(g)}+\left\|\nabla_{g}^{2} X^{\prime \prime}\right\|_{g}\right) \leq\left\|\nabla_{h}^{2} Y\right\|_{L^{2}(h)} \leq \Lambda_{k}\left(1+\epsilon^{\prime}\right)
$$

Since $\Lambda_{k}\|X\|_{L^{2}(g)}=\Lambda_{k}\left(1+O\left(\epsilon^{\prime}\right)\right)$ by the same argument as the above and $\left\|\nabla_{g}^{2} X^{\prime}\right\|_{L^{2}(g)}=$ $O\left(\epsilon^{\prime}\right)$, it follows that $\left\|\nabla_{g}^{2} X^{\prime \prime}\right\|_{g}=O\left(\epsilon^{\prime}\right)$.

Proposition 18. Let $h$ be a metric on $M$ such that $(1-\epsilon)\|g\|_{c^{2, \alpha}}<\|h\|_{c^{2, \alpha}}<(1+$ $\epsilon)\|g\|_{c^{2, \alpha}}$. There exist constants $\eta_{g, i}(\epsilon), 1 \leq i \leq J$, which go to zero when $\epsilon$ approaches zero, such that to any orthonormal basis $\left\{Y_{i}\right\}$ of eigenvector fields of $\nabla_{h}^{2}$ one can associate an orthonormal basis $\left\{X_{i}\right\}$ of eigenvector fields $\nabla_{g}^{2}$ satisfying $\left\|X_{i}-Y_{i}\right\|_{H^{1}(g)}+$ $\left\|\nabla_{g}^{2} X_{i}-\nabla_{g}^{2} Y_{i}\right\|_{L^{2}(g)} \leq \eta_{g, i}(\epsilon)$ for $i \leq J$.

Proof. Take any orthonormal basis $\left\{Y_{i}\right\}$ of eigenvector fields of $\nabla_{h}^{2}$. For each $k \leq k_{0}$ it defines an orthonormal basis of $F_{k}$, say $Y_{1}^{k}, \cdots, Y_{m_{k}}^{k}$. Applying the Gram-Schmidt process, we can orthonormalize the family $\pi_{k}\left(Y_{1}^{k}\right), \cdots, \pi_{k}\left(Y_{m_{k}}^{k}\right)$ of $E_{k}$ to an orthonormal basis $X_{1}^{k}, \cdots, X_{m_{k}}^{k}$. By Lemma 17 (a), $Y_{1}^{k}, \cdots, Y_{m_{k}}^{k}$ is $H^{1}(g)$-close to $\pi_{k}\left(Y_{1}^{k}\right), \cdots, \pi_{k}\left(Y_{m_{k}}^{k}\right)$. By Lemma 17 (b), the family $\pi_{k}\left(Y_{1}^{k}\right), \cdots, \pi_{k}\left(Y_{m_{k}}^{k}\right)$ is almost orthonormal and hence $H^{1}(g)$-close to $X_{1}^{k}, \cdots, X_{m_{k}}^{k}$. Thus we know that $Y_{1}^{k}, \cdots, Y_{m_{k}}^{k}$ is $H^{1}(g)$-close to $X_{1}^{k}, \cdots, X_{m_{k}}^{k}$. By the same argument, $\nabla_{g}^{2} Y_{1}^{k}, \cdots, \nabla_{g}^{2} Y_{m_{k}}^{k}$ and $\nabla_{g}^{2} X_{1}^{k}, \cdots, \nabla_{g}^{2} X_{m_{k}}^{k}$ are $L^{2}(g)$-close. This proves the Proposition.

To prove Theorem 16, we have to control the $L^{p}$ norm of the Hessian of a given vector field, and we need a Calderon-Zygmund type inequality, which is based the work of C. Wang [41].

Theorem 19. [41, Theorem 0.1] Let $\left(M^{n}, g\right), n \geq 3$, be a compact, connected, oriented Riemannian manifold without boundary. Suppose that

$$
\operatorname{inj}(M) \geq i_{0}, \operatorname{Vol}(M) \leq V, \text { and }|R i c| \leq \kappa .
$$

For $1<q<\infty$, there exists a constant $C_{q}=C\left(q, n, K, i_{0}, V\right)$, such that for any 1 -form $\phi \in H^{\perp}$,

$$
\|\operatorname{Hess} \phi\|_{L^{q}(g)} \leq C_{q}\left\|\Delta_{H} \phi\right\|_{L^{q}(g)}
$$

where $H$ is the space of harmonic 1-forms and $\Delta_{H}$ is the Hodge Laplacian.

We are now ready to prove Theorem 16 .

Proof. For $p>n$, by the Sobolev inequality [28] we have

$$
\|Q\|_{C^{1, \alpha}(g)} \leq C_{p}^{(1)}(g)\left(\left\|\operatorname{Hess}_{g} Q\right\|_{L^{p}(g)}+\left\|\nabla_{g} Q\right\|_{L^{p}(g)}+\|Q\|_{L^{p}(g)}\right)
$$


for any $Q \in C^{\infty}(T M)$. Note that vector fields are dual to 1-forms and the Hodge Laplacian and the connection Laplacian only differ by the the Ricci curvature due to the Weitzeonböck formula. By Theorem 19, for $W \in C^{\infty}(T M) \cap H^{\perp}$, we have

$$
\left\|\operatorname{Hess}_{g} W\right\|_{L^{p}(g)} \leq C\left(\left\|\nabla_{g}^{2} W\right\|_{L^{p}(g)}+\|W\|_{L^{p}(g)}\right)
$$

and hence

$$
\|W\|_{C^{1, \alpha}(g)} \leq C_{p}^{(2)}(g)\left(\left\|\nabla_{g}^{2} W\right\|_{L^{p}(g)}+\left\|\nabla_{g} W\right\|_{L^{p}(g)}+\|W\|_{L^{p}(g)}\right) .
$$

Let $\left\{Y_{i}\right\}$ be an orthonormal basis of eigenvector fields of $\nabla_{h}^{2}$ and let $\left\{X_{i}\right\}$ be the orthonormal basis of eigenvector fields of $\nabla_{g}^{2}$ associated with $\left\{Y_{i}\right\}$ given by Proposition 18. Denote $\|\cdot\|_{\infty}:=\|\cdot\|_{L^{\infty}(g)}$ to be the sup-norm with respect to $g$. Denote $\pi_{0}\left(X_{i}-Y_{i}\right)$ to be the harmonic part of $X_{i}-Y_{i}$ with respect to $g$, and $\pi_{0}^{\perp}\left(X_{i}-Y_{i}\right):=X_{i}-Y_{i}-$ $\pi_{0}\left(X_{i}-Y_{i}\right)=X_{i}-\left(Y_{i}-\pi_{0}\left(Y_{i}\right)\right)$. Clearly $\pi_{0}\left(X_{i}-Y_{i}\right)=-\pi_{0}\left(Y_{i}\right)=-\left\langle X_{0}, Y_{i}\right\rangle_{g} X_{0}$. Thus,

$$
\left\|X_{i}-Y_{i}\right\|_{C^{1, \alpha}(g)} \leq\left\|\pi_{0}\left(Y_{i}\right)\right\|_{C^{1, \alpha}(g)}+\left\|X_{i}-\pi_{0}^{\perp}\left(Y_{i}\right)\right\|_{C^{1, \alpha}(g)} .
$$

By Proposition 8 and Lemma 17(b), we immediately have that

$$
\left\|\pi_{0}\left(Y_{i}\right)\right\|_{C^{1, \alpha}(g)} \leq C\left\|\pi_{0}\left(Y_{i}\right)\right\|_{L^{2}(g)}=\left\|\left\langle X_{0}, Y_{i}\right\rangle_{g} X_{0}\right\|_{L^{2}(g)} \leq \beta_{k}(\epsilon) .
$$

By the Sobolev embedding,

$$
\begin{aligned}
& \left\|X_{i}-\pi_{0}^{\perp}\left(Y_{i}\right)\right\|_{C^{1, \alpha}(g)} \\
\leq & C_{p}^{(1)}(g)\left(\left\|\nabla_{g}^{2}\left(X_{i}-\pi_{0}^{\perp}\left(Y_{i}\right)\right)\right\|_{L^{p}(g)}+\left\|\nabla_{g}\left(X_{i}-\pi_{0}^{\perp}\left(Y_{i}\right)\right)\right\|_{L^{p}(g)}+\left\|X_{i}-\pi_{0}^{\perp}\left(Y_{i}\right)\right\|_{L^{p}(g)}\right) \\
\leq & C_{p}^{(2)}(g)\left(\left\|\nabla_{g}^{2}\left(X_{i}-\pi_{0}^{\perp}\left(Y_{i}\right)\right)\right\|_{L^{2}(g)}+\left\|X_{i}-\pi_{0}^{\perp}\left(Y_{i}\right)\right\|_{H^{1}(g)}\right)^{2 / p} \\
& \times\left(\left\|X_{i}\right\|_{\infty}+\left\|\pi_{0}^{\perp}\left(Y_{i}\right)\right\|_{\infty}+\left\|\nabla_{g} X_{i}\right\|_{\infty}+\left\|\nabla_{g} \pi_{0}^{\perp}\left(Y_{i}\right)\right\|_{\infty}+\left\|\nabla_{g}^{2} X_{i}\right\|_{\infty}+\left\|\nabla_{g}^{2} \pi_{0}^{\perp}\left(Y_{i}\right)\right\|_{\infty}\right)(p-2) / p
\end{aligned}
$$

where the last inequality follows by Hölder's inequality that

$$
\left\|\nabla_{g}^{2}\left(X_{i}-\pi_{0}^{\perp}\left(Y_{i}\right)\right)\right\|_{L^{p}(g)} \leq\left\|\nabla_{g}^{2}\left(X_{i}-\pi_{0}^{\perp}\left(Y_{i}\right)\right)\right\|_{L^{2}(g)}^{2 / p}\left\|\nabla_{g}^{2}\left(X_{i}-\pi_{0}^{\perp}\left(Y_{i}\right)\right)\right\|_{\infty}^{(p-2) / p}
$$

and

$$
\begin{aligned}
& \left\|\nabla_{g}\left(X_{i}-\pi_{0}^{\perp}\left(Y_{i}\right)\right)\right\|_{L^{p}(g)}+\left\|X_{i}-\pi_{0}^{\perp}\left(Y_{i}\right)\right\|_{L^{p}(g)} \\
\leq & C\left\|X_{i}-\pi_{0}^{\perp}\left(Y_{i}\right)\right\|_{H^{1}(g)}^{2 / p}\left(\left\|X_{i}-\pi_{0}^{\perp}\left(Y_{i}\right)\right\|_{\infty}+\left\|\nabla_{g} X_{i}-\nabla_{g} \pi_{0}^{\perp}\left(Y_{i}\right)\right\|_{\infty}\right)^{(p-2) / p} .
\end{aligned}
$$

By the triangular inequality we have

$$
\begin{aligned}
& \left\|\nabla_{g}^{2}\left(X_{i}-\pi_{0}^{\perp}\left(Y_{i}\right)\right)\right\|_{L^{2}(g)} \\
\leq & \left\|\nabla_{g}^{2}\left(X_{i}-Y_{i}\right)\right\|_{L^{2}(g)}+\left\|\nabla_{g}^{2} \pi_{0}\left(Y_{i}\right)\right\|_{L^{2}(g)}=\left\|\nabla_{g}^{2}\left(X_{i}-Y_{i}\right)\right\|_{L^{2}(g)}
\end{aligned}
$$

since $\nabla_{g}^{2} \pi_{0}\left(Y_{i}\right)=0$ and

$$
\left\|X_{i}-\pi_{0}^{\perp}\left(Y_{i}\right)\right\|_{H^{1}(g)} \leq\left\|X_{i}-Y_{i}\right\|_{H^{1}(g)}+\left\|\pi_{0}\left(Y_{i}\right)\right\|_{L^{2}(g)}
$$

where we use the fact that $\left\|\pi_{0}\left(Y_{i}\right)\right\|_{H^{1}(g)}=\left\|\pi_{0}\left(Y_{i}\right)\right\|_{L^{2}(g)}$ since $\nabla_{g} \pi_{0}\left(Y_{i}\right)=0$. Further, note that

$$
\left\|\pi_{0}^{\perp}\left(Y_{i}\right)\right\|_{\infty} \leq\left\|Y_{i}\right\|_{\infty}+\left\|\pi_{0}\left(Y_{i}\right)\right\|_{\infty} \leq\left\|Y_{i}\right\|_{\infty}+\beta_{k}(\epsilon)
$$


by (5.18). Similarly, we have

$$
\left\|\nabla_{g} \pi_{0}^{\perp}\left(Y_{i}\right)\right\|_{\infty} \leq\left\|\nabla_{g} Y_{i}\right\|_{\infty}+\left\|\nabla_{g} \pi_{0}\left(Y_{i}\right)\right\|_{\infty}=\left\|\nabla_{g} Y_{i}\right\|_{\infty}
$$

and

$$
\left\|\nabla_{g}^{2} \pi_{0}^{\perp}\left(Y_{i}\right)\right\|_{\infty} \leq\left\|\nabla_{g}^{2} Y_{i}\right\|_{\infty}+\left\|\nabla_{g}^{2} \pi_{0}\left(Y_{i}\right)\right\|_{\infty}=\left\|\nabla_{g}^{2} Y_{i}\right\|_{\infty}
$$

since $\nabla_{g} \pi_{0}\left(Y_{i}\right)=\nabla_{g}^{2} \pi_{0}\left(Y_{i}\right)=0$.

By plugging (5.22), (5.23), (5.24), (5.25) and (5.26) into (5.19), together with (5.18), (5.17) becomes

$$
\begin{aligned}
& \left\|X_{i}-Y_{i}\right\|_{C^{1, \alpha}(g)} \\
\leq & C_{p}^{(2)}(g)\left(\left\|\nabla_{g}^{2}\left(X_{i}-Y_{i}\right)\right\|_{L^{2}(g)}+\left\|X_{i}-Y_{i}\right\|_{H^{1}(g)}+\beta_{k}(\epsilon)\right)^{2 / p} \\
& \times\left(\left\|X_{i}\right\|_{\infty}+\left\|Y_{i}\right\|_{\infty}+\left\|\nabla_{g} X_{i}\right\|_{\infty}+\left\|\nabla_{g} Y_{i}\right\|_{\infty}+\left\|\nabla_{g}^{2} X_{i}\right\|_{\infty}+\left\|\nabla_{g}^{2} Y_{i}\right\|_{\infty}+\beta_{k}(\epsilon)\right)^{(p-2) / p} .
\end{aligned}
$$

Note that by Proposition 18 , for all $1 \leq i<k_{0}$ there exist constants $\eta_{g, j}(\epsilon)$ such that

$$
\left\|X_{i}-Y_{i}\right\|_{H^{1}(g)}+\left\|\nabla_{g}^{2}\left(X_{i}-Y_{i}\right)\right\|_{L^{2}(g)} \leq \eta_{g, j}(\epsilon) .
$$

Further, since the metric $h$ is $\epsilon$ close to $g$ in $c^{2, \alpha}$, the norms we consider for either the metric $g$ or the metric $h$ are comparable. Also note that $\left\|\nabla_{g}^{2} X_{i}\right\|_{L^{\infty}(g)}=\lambda_{i}(g)\left\|X_{i}\right\|_{L^{\infty}(g)} \leq$ $C\left\|X_{i}\right\|_{L^{\infty}(g)}$ and $\left\|\nabla_{h}^{2} Y_{i}\right\|_{L^{\infty}(h)}=\lambda_{i}(h)\left\|Y_{i}\right\|_{L^{\infty}(h)} \leq C\left\|Y_{i}\right\|_{L^{\infty}(h)}$. Therefore, to complete the proof, it suffices to give uniform bounds for $\left\|X_{i}\right\|_{L^{\infty}(g)},\left\|Y_{i}\right\|_{L^{\infty}(h)},\left\|\nabla_{g} X_{i}\right\|_{L^{\infty}(g)}$, and $\left\|\nabla_{h} Y_{i}\right\|_{L^{\infty}(h)}$ for $i \leq k_{0}$.

By the assumption on the injectivity and volume, there exists an universal number $N_{0}$ so that $(M, g)$ and $(M, h)$ are covered by $N_{0}$-balls. Indeed, due to the lower bound of the injectivity, by Croke's lemma [14], the balls of radius $\epsilon$ is bounded from below; on the other hand, since the volume is bounded by $V$, we have a bounded number of balls. Thus, by applying Proposition 8 , there exists some constant $C=C\left(i_{0}, V, \alpha, n, Q, \kappa, k_{0}\right)$ so that

$$
\left\|X_{i}\right\|_{L^{\infty}(g)},\left\|Y_{i}\right\|_{L^{\infty}(h)},\left\|\nabla_{g} X_{i}\right\|_{L^{\infty}(g)}, \text { and }\left\|\nabla_{h} Y_{i}\right\|_{L^{\infty}(h)} \leq C
$$

for $i \leq k_{0}$.

\section{EXAMPLES}

In this section, we illustrate how VDM is carried out numerically. A detailed discussion of the algorithm and its application to different problems could be found in [33, 45, 11, 1, 25], and its robustness analysis could be found in [19]

Suppose we sampled $n$ points $\mathcal{X}:=\left\{x_{i}\right\}_{i=1}^{m}$ uniformly from a $n$-dim smooth manifold $M$ without boundary embedded in $\mathbb{R}^{p}$ via $\iota$; that is, $\left\{x_{i}\right\}_{i=1}^{m} \subset \iota(M) \subset \mathbb{R}^{p}$. Assume that $M$ is endowed with a Riemannian metric $g$ induced from the ambient Euclidean space via $\iota$. We construct the graph connection Laplacian (GCL) $C \in \mathbb{R}^{m n \times m n}$ in the following way.

- Step 1: build up an undirected graph $G=(V, E)$, where $V=\mathcal{X}$ and $E$ is the edges $E=\left\{\left(x_{i}, x_{j}\right) \mid i \neq j\right\}$. Note that there are different ways to design $E$ from a given point cloud. To demonstrate the idea, here we simply design the edge so that the undirected graph is complete. 
- Step 2: establish the affinity function, $w: E \rightarrow \mathbb{R}^{+}$, by setting $w\left(x_{i}, x_{j}\right)=$ $K\left(\left\|x_{i}-x_{j}\right\|_{\mathbb{R}^{p}} / \sqrt{\epsilon}\right)$, where $\epsilon>0$ is the "bandwidth" and $K$ is a suitable kernel function chosen by the user. In all experiments in this section, we used the kernel function $K(u)=e^{-5 u^{2}} \chi_{[0,1]}$ for the definition of the weight function $w$.

- Step 3: for $x_{i}$, choose a basis $\left\{u_{i, 1}, \ldots, u_{i, n}\right\} \subset \mathbb{R}^{p}$, of the embedded tangent space, $\iota_{*} T_{x_{i}} M$, which is a $d$-dim affine subspace in $\mathbb{R}^{p}$. Denote $B_{i}=$ $\left[u_{i, 1}, \ldots, u_{i, n}\right] \in \mathbb{R}^{p \times n}$. In general, we do not have the information of $\iota_{*} T_{x_{i}} M$, and need to estimate $B_{i}$ via the local principal component analysis based on the fact that locally the manifold could be well approximated by the embedded tangent space [33].

- Step 3: establish the connection function, $g: E \rightarrow O(d)$, where $g\left(x_{i}, x_{j}\right)=O_{i j}$ and $O_{i j}=\arg \min _{O \in S O(n)}\left\|B_{i}^{T} B_{j}-O\right\|$. Here $O_{i j}$ is an approximation of the parallel transport from $x_{j}$ to $x_{i}$ associated with the induced metric $g$ [33]. Note that in general, due to the curvature, $B_{i}^{T} B_{j}$ is not an orthogonal matrix, so we need an optimization step to obtain the parallel transport.

- Step 4: establish a $n \times n$ block matrix $S$, with $d \times d$ block entries, so that the $(i, j)$-th block is $S[i, j]=w\left(x_{i}, x_{j}\right) g\left(x_{i}, x_{j}\right)$; establish a $n \times n$ diagonal block matrix $D$, with $d \times d$ block entries, so that the $(i, j)$-th block is $D[i, j]=$ $\sum_{l=1}^{m} w\left(x_{i}, x_{l}\right) I_{n \times n} \delta_{i j}$

- Step 5: establish the GCL $C=I_{m n \times m n}-D^{-1} S$.

With the GCL $C$, we could evaluate its eigenvalue $\lambda_{l}$ and eigenvectors $u_{l} \in \mathbb{R}^{m n}$, and order the eigenvalues in the increasing order: $\lambda_{1} \leq \lambda_{2} \leq \ldots$. Denote $u_{i}[l] \in \mathbb{R}^{n}$ as $u_{i}[l]=\left(u_{i}((l-1) d+1), \ldots, u_{i}(\ln )\right)^{T}$. The VDM with the diffusion time $t>0$ for the sampled point cloud $\left\{x_{i}\right\}_{i=1}^{n}$ is defined as

$$
V_{t}: x_{i} \mapsto\left(e^{-\left(\lambda_{k}+\lambda_{l}\right) t}\left\langle u_{k}[i], u_{l}[j]\right\rangle\right)_{l, k=1}^{m n} \subset \mathbb{R}^{(m n)^{2}} .
$$

Here, we emphasize that $u_{i}[l]$ is actually the coordinate of the $i$-th eigenvector field at $x_{l}$ associated with the basis chosen for $T_{x_{l}} M$ [36]. It has been shown that asymptotically, the GCL will converge not only pointwisely but also spectrally to the connection Laplacian of $M$. Thus, we could numerically implement VDM. Note that in general we could not visualize a geometric object with dimension higher than 3, so in the following numerical implementation, we illustrate the truncated VDM by choosing suitable subset of embedding coordinates.

The first example is taking the manifold $M$ to be diffeomorphic to $S^{1}$ embedded in $\mathbb{R}^{p}$. Here, $M$ is generated by the X-ray transform of a 2-dim image compactly supported on $\mathbb{R}^{2}$. The dataset is generated in the following way. Take the image of interest to be a compactly supported function $f$ so that $\operatorname{supp}(f) \subset B_{1}$, where $B_{1}$ is the ball with center 0 and radius 1 . Sample uniformly $m$ points on $S^{1}$, denoted as $\left\{\theta_{i}\right\}_{i=1}^{m}$. The dataset is generated by discretizing the X-ray transform by $x_{i}:=\left[R_{\psi}\left(\theta_{i}, z_{j}\right)\right]_{i=1}^{p}$, where $i=1, \ldots, n, R_{\psi}\left(\theta_{i}, z_{j}\right)=\int \psi\left(y \theta_{i}+z_{j}\right) d y, z_{j}=-1+2 j / p$ and $p$ is chosen big enough so that the topology of $R_{\psi}\left(S^{1}\right)$ is preserved after discretization. Note that while we sample uniformly from $S^{1}$, after the X-ray transform $R_{\psi}$, which is a diffeomorphism from $S^{1}$ to a 1-dim manifold $M$, the dataset $\left\{x_{i}\right\}_{i=1}^{m}$ is non-uniformly sampled from $M$. We refer the reader with interest to [34] for more details about this 2-dim random 
tomography problem. Take $m=2000$. Note that since the tangent bundle of $M$, which is diffeomorphic to $S^{1}$, is trivial, the connection Laplacian is reduced to the Laplace-Beltrami operator. Thus, the first eigenvector field is the constant function, the second and third eigenvector field are the sine and cosine functions, and so on. We thus consider the following truncated VDM

$$
V_{1}^{(2)}: x_{i} \mapsto\left(e^{-\left(\lambda_{1}+\lambda_{2}\right)}\left\langle u_{1}[i], u_{2}[i]\right\rangle, e^{-\left(\lambda_{1}+\lambda_{3}\right)}\left\langle u_{1}[i], u_{3}[i]\right\rangle\right)^{T} \in \mathbb{R}^{2},
$$

which embeds $M$ into $\mathbb{R}^{2}$. Figure 6.1 shows this truncated VDM. Note VDM generates a non-canonical circle embedded in $\mathbb{R}^{2}$, due to the non-uniform distribution on $M$.

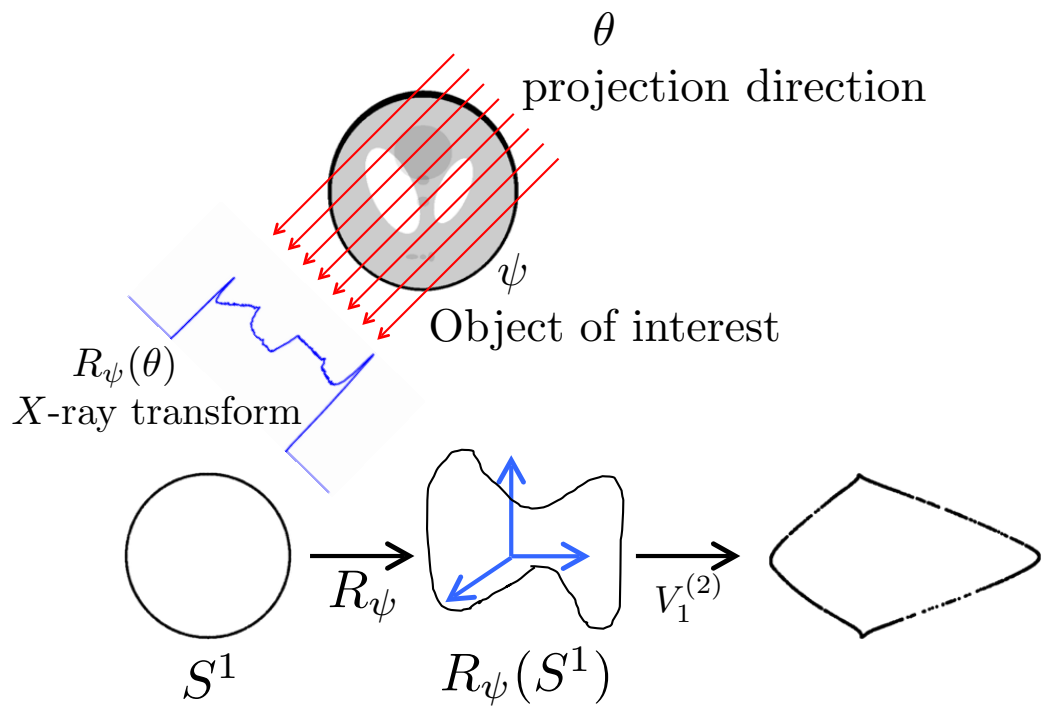

Figure 6.1. A nontrivial $S^{1}$ generated by the X-ray transform and the VDM result.

The second example shows that we might not be able to visualize $S^{2}$ by VDM. Take the manifold to be the canonical $S^{2}$ embedded in $\mathbb{R}^{3}$. Take $m=2000$. Note that although the tangent bundle of $S^{2}$ is nontrivial, its well-known that the first few eigenvector fields could be easily evaluated - the eigenvector fields associated with the smallest eigenvalue, $\lambda_{1}$, of the connection Laplacian, denoted as $X_{1}, X_{2}$ and $X_{3}$, are nothing but the project of the constant vector fields $(1,0,0),(0,1,0)$ and $(0,0,1)$ on $\mathbb{R}^{3}$ onto the embedded tangent space. Also, since $S^{2}$ is a Riemannian surface with the complex structure, the "complex conjugate" of $X_{1}$ (respectively $X_{2}$ and $X_{3}$ ), denoted as $X_{4}$ (respectively $X_{5}$ and $X_{6}$ ), is also an eigenvector field associated with $\lambda_{1}$. We carry out the following calculation to explicitly evaluate these eigenvector fields. Denote

$$
R=\left[\begin{array}{ccc}
\mid & \mid & \mid \\
R^{1} & R^{2} & R^{3} \\
\mid & \mid & \mid
\end{array}\right]=\left[\begin{array}{ccc}
R_{1}^{1} & R_{1}^{2} & R_{1}^{3} \\
R_{2}^{1} & R_{2}^{2} & R_{2}^{3} \\
R_{3}^{1} & R_{3}^{2} & R_{3}^{3}
\end{array}\right] \in S O(3) .
$$


Since $S O(3)$ is the frame bundle of $S^{2}$, if we view $R^{3} \in S^{2}$ as a sampled point from $S^{2}, R^{1}$ and $R^{2}$ form a basis of the embedded tangent plane of $T_{R^{3}} S^{2}$, which is an affine subspace of $\mathbb{R}^{3}$. By a direct calculation, we know that $\iota_{*} X_{1}$, the embedded vector field $X_{1}$ in $\mathbb{R}^{3}$, becomes

$$
\iota_{*} X_{1}\left(R^{3}\right)=\left[\begin{array}{cc}
\mid & \mid \\
R^{1} & R^{2} \\
\mid & \mid
\end{array}\right]\left[\begin{array}{cc}
\mid & \mid \\
R^{1} & R^{2} \\
\mid & \mid
\end{array}\right]^{T}\left[\begin{array}{l}
1 \\
0 \\
0
\end{array}\right]=\left[\begin{array}{cc}
\mid & \mid \\
R^{1} & R^{2} \\
\mid & \mid
\end{array}\right]\left[\begin{array}{l}
R_{1}^{1} \\
R_{1}^{2}
\end{array}\right] .
$$

Similarly, for $k=2,3$, we know that

$$
\iota_{*} X_{k}\left(R^{3}\right)=\left[\begin{array}{cc}
\mid & \mid \\
R^{1} & R^{2} \\
\mid & \mid
\end{array}\right]\left[\begin{array}{l}
R_{k}^{1} \\
R_{k}^{2}
\end{array}\right] .
$$

By the cross product associated with the almost complex structure on $S^{2}, X_{4}, X_{5}$ and $X_{6}$ could be evaluated directly. For $k=1,2,3$, we have

$$
\iota_{*} X_{k+3}\left(R^{3}\right)=R^{3} \times \iota_{*} X_{k}\left(R^{3}\right)=\left[\begin{array}{cc}
\mid & \mid \\
R^{2} & -R^{1} \\
\mid & \mid
\end{array}\right]\left[\begin{array}{l}
R_{k}^{1} \\
R_{k}^{2}
\end{array}\right]=R_{k}^{1} R^{2}-R_{k}^{2} R^{1} .
$$

Thus,

$$
\begin{aligned}
\left\langle X_{k}\left(R^{3}\right), X_{l}\left(R^{3}\right)\right\rangle & =\left\langle X_{k+3}\left(R^{3}\right), X_{l+3}\left(R^{3}\right)\right\rangle=R_{k}^{1} R_{l}^{1}+R_{k}^{2} R_{l}^{2}, \\
\left\langle X_{k}\left(R^{3}\right), X_{l+3}\left(R^{3}\right)\right\rangle & =-R_{k}^{1} R_{l}^{2}+R_{k}^{2} R_{l}^{1},
\end{aligned}
$$

for $k, l=1, \ldots, 3$. By a direct calculation, we have $\left\langle X_{k}\left(R^{3}\right), X_{l}\left(R^{3}\right)\right\rangle=\left\langle X_{k}\left(-R^{3}\right), X_{l}\left(-R^{3}\right)\right\rangle$ and $\left\langle X_{k}\left(R^{3}\right), X_{l+3}\left(R^{3}\right)\right\rangle=-\left\langle X_{k}\left(-R^{3}\right), X_{l+3}\left(-R^{3}\right)\right\rangle$. Further, when $k \neq l$, the map $R^{3} \mapsto\left\langle X_{k}\left(R^{3}\right), X_{l+3}\left(R^{3}\right)\right\rangle$ maps $\pm e_{k}$ and $\pm e_{l}$ to 0 . Thus, the truncated VDM depending only on a randomly chosen three $\left\langle X_{k}\left(R^{3}\right), X_{l}\left(R^{3}\right)\right\rangle, k, l=1, \ldots, 6$, is not an embedding. Note that this is different from the diffusion maps - we could simply visualize $S^{2}$ via the truncated diffusion maps by taking the top three eigenfunctions into account. Also, we know that the range of the map

$$
V_{1}^{(3)}: x_{i} \mapsto\left(e^{-2 \lambda_{k}}\left\langle u_{k}[i], u_{k}[i]\right\rangle\right)_{k=1}^{3} \in \mathbb{R}^{3}
$$

is diffeomorphic to $\mathbb{R} P^{2}$.

The theoretical analysis and the numerical results open the following questions. Although the theorem shows that each compact smooth manifold without boundary could be embedded to a finite dimensional Euclidean space by the truncated VDM taking finite eigenvector fields into account, the number of necessary eigenvector fields is not clear. The same problem happens when we discuss the spectral embedding by the truncated diffusion map. Even worse, while the purpose of many nonlinear embedding algorithms is to "reduce the dimension", there is no guarantee that we could achieve it if we could not know the necessary number of the eigenvector fields. Recall that by "reduce the dimension", we mean that the "dimension" of the sampled point cloud could be well represented by another point cloud embedded in a lower dimensional space. Without the knowledge of the necessary number of eigenvector fields, we could 
not rule out the possibility that the number of eigenvector fields we need for the truncated VDM (or the number of eigenfunctions in the truncated DM) might be more than the ambient space dimension of the original dataset. We thus need to find a way to estimate the necessary number of the eigenvector fields (respectively eigenfunctions) for the truncated VDM (respectively DM). Ideally we would like to have a bound of this number based on the basic geometric/topological quantities. This problem is prevalent in the field actually - for a given nonlinear embedding algorithm, how to evaluate if the dimension reduction mission for a dataset could be achieved? To the best of our knowledge, this is also an open problem for most nonlinear embedding algorithm.

\section{REFERENCES}

[1] B. Alexeev, A. S. Bandeira, M. Fickus, and D. G. Mixon. Phase retrieval with polarization. SIAM J. Imaging Sci., 2013.

[2] M. Anderson. Convergence and rigidity of manifolds under ricci curvature bounds. Inventiones mathematicae, 102:429-445, 1990.

[3] M. T. Anderson and J. Cheeger. $c^{\alpha}$-compactness for manifolds with ricci curvature and injectivity radius bounded below. J. Differential Geometry, 35:265-281, 1992.

[4] J. Bates. The embedding dimension of laplacian eigenfunction maps. Appl. Comput. Harmon. Anal., 37(3):516-530, 2014.

[5] M. Belkin and P. Niyogi. Laplacian Eigenmaps for Dimensionality Reduction and Data Representation. Neural. Comput., 15(6):1373-1396, June 2003.

[6] P. Bérard. Spectral Geometry: Direct and Inverse Problems. Springer, 1986.

[7] P. Bérard, G. Besson, and S. Gallot. Embedding riemannian manifolds by their heat kernel. Geom. Funct. Anal., 4:373-398, 1994. 10.1007/BF01896401.

[8] N. Berline, E. Getzler, and M. Vergne. Heat kernels and Dirac operators. Grundlehren Text Editions. Springer, 2004.

[9] B. Chow, P. Lu, and L. Ni. Hamilton's Ricci Flow. American Mathematical Society, 2006.

[10] F. Chung. Spectral Graph Theory. American Mathematical Society, 1996.

[11] F. Chung and M. Kempton. A local clustering algorithm for connection graphs. In Anthony Bonato, Michael Mitzenmacher, and Pawel Pralat, editors, Algorithms and Models for the Web Graph, volume 8305 of Lecture Notes in Computer Science, pages 26-43. Springer International Publishing, 2013.

[12] F. Chung, W. Zhao, and M. Kempton. Ranking and sparsifying a connection graph. In Anthony Bonato and Jeannette Janssen, editors, Algorithms and Models for the Web Graph, volume 7323 of Lecture Notes in Computer Science, pages 66-77. Springer Berlin Heidelberg, 2012.

[13] R. R. Coifman and S. Lafon. Diffusion maps. Appl. Comput. Harmon. Anal., 21(1):5-30, 2006.

[14] C. Croke. Some isoperimetric inequalities and eigenvalue estimates. Ann. Sci. Ecole Norm. Sup., 13(419-435), 1980.

[15] D. DeTurck and J. Kazdan. Some regularity theorems in riemannian geometry. Ann. Scient. Ec. Norm. Sup., 14:249-260, 1981.

[16] G. Dolzmann and S. M'uller. Estimates for green's matrices of elliptic systems by $l^{p}$ theory. Manuscripta Mathematica, pages 261-273, 1995.

[17] D. L. Donoho and C. Grimes. Hessian eigenmaps: Locally linear embedding techniques for highdimensional data. P. Natl. Acad. Sci. USA, 100(10):5591-5596, 2003.

[18] N. El Karoui and H.-T. Wu. Connection graph Laplacian and random matrices with random blocks. Information and Inference: A Journal of the IMA, 4:1-42, 2015.

[19] N. El Karoui and H.-T. Wu. Connection graph Laplacian methods can be made robust to noise. Ann. Stat., 2015. Accepted for Publication.

[20] P. Gilkey. The Index Theorem and the Heat Equation. Boston : Publish or Perish, inc., 1974. 
[21] R. Hadani and A. Singer. Representation theoretic patterns in three dimensional Cryo-Electron Microscopy I: The intrinsic reconstitution algorithm. Annals of Mathematics, 174(2):1219-1241, 2011.

[22] P. Jones, M. Maggioni, and R. Schul. Universal local parametrization via heat kernels and eigenfunctions of the laplacian. Ann. Acad. Sci. Fenn. Math., 35:131-174, 2010.

[23] R. R. Lederman and R. Talmon. Learning the geometry of common latent variables using alternating-diffusion. Appl. Comput. Harmon. Anal., In press, 2015.

[24] P. Li and S. T. Yau. On the parabolic kernel of the schrödinger operator. Acta Mathematica, 156(1):153-201, 1986.

[25] S. Marchesini, Y.-C. Tu, and H.-T. Wu. Alternating Projection, Ptychographic Imaging and Phase Synchronization. Appl. Comput. Harmon. Anal., 2015. In press. arXiv:1402.0550 [math.OC].

[26] C.B. Morrey. Multiple Integrals in the Calculus of Variateions. Springer, 1966.

[27] J. Nash. The Imbedding Problem for Riemannian Manifolds. Annals of Mathematics, 63(1):20-63, 1956.

[28] R. Palais. Foundations of Global Non-Linear Analysis. W. A. Benjamin, 1968.

[29] J. W. Portegies. Embeddings of Riemannian manifolds with heat kernels and eigenfunctions. Comm. Pure Appl. Math., 2015. arXiv:1311.7568 [math.DG].

[30] S. T. Roweis and L. K. Saul. Nonlinear dimensionality reduction by locally linear embedding. Science, 290(5500):2323-2326, 2000.

[31] A. Singer and R. R. Coifman. Non-linear independent component analysis with diffusion maps. Appl. Comput. Harmon. Anal., 25(2):226 - 239, 2008.

[32] A. Singer and H.-T. Wu. Orientability and diffusion map. Appl. Comput. Harmon. Anal., 31(1):44$58,2011$.

[33] A. Singer and H.-T. Wu. Vector diffusion maps and the connection Laplacian. Comm. Pure Appl. Math., 65(8):1067-1144, 2012.

[34] A. Singer and H.-T. Wu. 2-d tomography from noisy projections taken at unknown random directions. SIAM J. Imaging Sci., 6(1):136-175, 2013.

[35] A. Singer and H.-T. Wu. Spectral convergence of the connection laplacian from random samples. submitted, 2014.

[36] A. Singer and H.-T. Wu. Spectral convergence of the connection laplacian from random samples. Information and Inference: A Journal of the IMA, Accepted for publication, 2016.

[37] A. Singer, Zhao Z., Shkolnisky Y., and Hadani R. Viewing angle classification of cryo-electron microscopy images using eigenvectors. SIAM J. Imaging Sci., 4(2):723-759, 2011.

[38] R. Talmon and R. R. Coifman. Empirical Intrinsic Geometry for Intrinsic Modeling and Nonlinear Filtering. Proc. Nat. Acad. Sci., 110(31):12535-12540, 2013.

[39] R. Talmon and H.-T. Wu. Discovering a latent common manifold with alternating diffusion for multimodal sensor data analysis. submitted, 2015.

[40] J. B. Tenenbaum, V. de Silva, and J. C. Langford. A Global Geometric Framework for Nonlinear Dimensionality Reduction. Science, 290(5500):2319-2323, 2000.

[41] C. Wang. The calderón-zygmund inequality on a compact riemannian manifold. Pacific Journal of Mathematics, 217(1):181-200, 2004.

[42] X. Wang and K. Zhu. Isometric Embeddings via Heat Kernel. J. Differential Geometry, 99:497$538,2015$.

[43] H Whitney. The singularities of a smooth n-manifold in (2n-1)-space. Ann. of Math, 45:247-293, 1944.

[44] H.-T. Wu. Embedding riemannian manifolds by the heat kernel of the connection laplacian. submitted, 2014.

[45] Z. Zhao and A. Singer. Rotationally Invariant Image Representation for Viewing Direction Classification in Cryo-EM. Journal of Structural Biology, 186(1):153-166, 2014. 
Chen-Yun Lin, Department of Mathematics, University of Toronto E-mail address: cylin@math.toronto.edu

Hau-Tieng Wu, Department of Mathematics, University of Toronto

E-mail address: hauwu@math.toronto.edu 